\title{
LINEAR ADDITIVE FUNCTIONALS OF SUPERDIFFUSIONS AND RELATED NONLINEAR P.D.E.
}

\author{
E. B. DYNKIN AND S. E. KUZNETSOV
}

\begin{abstract}
Let $L$ be a second order elliptic differential operator in a bounded smooth domain $D$ in $\mathbb{R}^{d}$ and let $1<\alpha \leq 2$. We get necessary and sufficient conditions on measures $\eta, \nu$ under which there exists a positive solution of the boundary value problem

$$
\begin{gathered}
-L v+v^{\alpha}=\eta \quad \text { in } D \\
v=\nu \quad \text { on } \partial D .
\end{gathered}
$$

The conditions are stated both analytically (in terms of capacities related to the Green's and Poisson kernels) and probabilistically (in terms of branching measure-valued processes called ( $L, \alpha)$-superdiffusions).

We also investigate a closely related subject - linear additive functionals of superdiffusions. For a superdiffusion in an arbitrary domain $E$ in $\mathbb{R}^{d}$, we establish a 1-1 correspondence between a class of such functionals and a class of $L$-excessive functions $h$ (which we describe in terms of their Martin integral representation). The Laplace transform of $A$ satisfies an integral equation which can be considered as a substitute for $(*)$.
\end{abstract}

\section{INTRODUCTION}

1.1. Boundary value problem with measures. We start from a differential operator

$$
L u=\sum_{i, j} a_{i j} \nabla_{i} \nabla_{j} u+\sum_{i} b_{i} \nabla_{i} u
$$

$\left(\nabla_{i}\right.$ stands for the partial derivative with respect to $\left.x_{i}\right)$ in a bounded smooth domain $D$ of $\mathbb{R}^{d}$ with coefficients subject to conditions:

1.1.A. (Uniform ellipticity) There exists a constant $\varkappa>0$ such that

$$
\sum_{i, j} a_{i j} \lambda_{i} \lambda_{j} \geq \varkappa \sum_{i} \lambda_{i}^{2} \quad \text { for all } x \in D, \lambda_{1}, \ldots, \lambda_{d} \in \mathbb{R}
$$

$$
\text { 1.1.B. } a_{i j} \in C^{2, \lambda}(\bar{D}), b_{i} \in C^{1, \lambda}(\bar{D}) .^{1}
$$

\footnotetext{
Received by the editors March 29, 1995.

1991 Mathematics Subject Classification. Primary 60J60, 35J65; Secondary 60J80, 31C15, 60J25, 60J55, 31C45, 35J60.

Partially supported by National Science Foundation Grant DMS-9301315

${ }^{1}$ We follow standard notation in P.D.E. (see, e.g., [21]). [Smooth domain means a domain of class $C^{2, \lambda}$.]
} 
The classical boundary value problem

$$
\begin{gathered}
-L v+v^{\alpha}=\rho \quad \text { in } D, \\
v=\sigma \quad \text { on } \partial D
\end{gathered}
$$

(with Hölder continuous $\rho$ and continuous $\sigma$ ) is equivalent to an integral equation

$$
v(x)+\int_{D} g(x, y) v(y)^{\alpha} d y=h(x)
$$

where

$$
h(x)=\int_{D} g(x, y) \rho(y) d y+\int_{\partial D} k(x, y) \sigma(y) a(d y),
$$

$g(x, y)$ is Green's function, $k(x, y)$ is the Poisson kernel of $L$ in $D$ and $a(d y)$ is the surface area on $\partial D$. We interpret $v$ as a (generalized) solution of the problem

$$
\begin{gathered}
-L v+v^{\alpha}=\eta \quad \text { on } D, \\
v=\nu \quad \text { on } \partial D
\end{gathered}
$$

involving two measures $\eta$ and $\nu$ if the equation (1.3) holds with

$$
h(x)=\int_{D} g(x, y) \eta(d y)+\int_{\partial D} k(x, y) \nu(d y) .
$$

In Theorem 1.1, we establish sufficient conditions on $\eta$ and $\nu$ under which problem (1.5) has a solution. Necessary conditions are established in Theorem 1.2. The equivalence of both sets of conditions follows from results in [17]. [Theorems 1.1 and 1.2 are still valid if $D$ is not smooth. However, in general, the equivalence of conditions imposed on $\nu$ in the two theorems is not proved.]

Particular cases of problem (1.5) have been studied before. The case $\nu=0$ was treated in [2] and the case $\eta=0$ was considered in [18]. Even earlier, Gmira and Véron [22] have investigated a class of functions $\psi$ such that the problem

$$
\begin{aligned}
\Delta v=\psi(v) & \text { on } D, \\
v=\nu & \text { on } \partial D
\end{aligned}
$$

has a solution for every finite measure $\nu$. This class contains $\psi(v)=v^{\alpha}$ with $(\alpha+1) /(\alpha-1)>d$.

1.2. $L$-diffusions. Suppose $D$ is a bounded smooth domain and that $L$ satisfies conditions 1.1.A,B. Then there exists ${ }^{2}$ a strictly positive function $p_{t}(x, y), t>$ $0, x, y \in D$ such that:

1.2.A. If $f$ is a continuous function on $D$ with compact support and if

$$
u_{t}(x)=\int_{D} p_{t}(x, y) f(y) d y,
$$

\footnotetext{
${ }^{2}$ This is proved (under weaker conditions on $L$ ) in Chapter 1 of [19].
} 
then

$$
\begin{gathered}
\frac{\partial u_{t}(x)}{\partial t}=L u_{t}(x), \\
u_{t}(x) \rightarrow f(x) \quad \text { as } t \rightarrow 0
\end{gathered}
$$

and

$$
u_{t}(x) \rightarrow 0 \quad \text { as } x \rightarrow z \in \partial D .
$$

(All partial derivatives of $p$ which appear in (1.8) are continuous in $(t, x, y)$.)

Function $p_{t}(x, y)$ has the following properties:

1.2.B. For all $s, t>0, x, z \in D$,

$$
\int_{D} p_{s}(x, y) d y p_{t}(y, z)=p_{s+t}(x, z) .
$$

1.2.C. For all $t>0, x \in D$,

$$
\int_{D} p_{t}(x, y) d y \leq 1
$$

Therefore $p_{t}(x, d y)=p_{t}(x, y) d y$ is a Markov transition function. It is well-known (see, e.g., [6]) that there exists a continuous Markov process $\xi=\left(\xi_{t}, \Pi_{x}\right)$ in $D$ with this transition function. We call it an $L$-diffusion. If $\zeta$ is the life time of $\xi$, then $\xi_{\zeta-}$ belongs to $\partial D$. By setting $\xi_{t}=\xi_{\zeta-}$ for $t \geq \zeta$, we define an $L$-diffusion stopped at the exit from $D$. Note that $\zeta$ can be interpreted as the first exit time of this process from $D$; often we use the notation $\tau$ for it.

Now suppose that $E$ is an arbitrary domain in $\mathbb{R}^{d}$ and that $L$ is a differential operator in $E$ which satisfies conditions 1.1.A, B in each bounded domain $D$ with $\bar{D} \subset E$. Consider a sequence of bounded smooth domains $D_{n}$ such that $\bar{D}_{n} \subset D_{n+1}$ and $\bigcup D_{n}=E$. The corresponding functions $p_{t}^{n}(x, y)$ increase monotonically and they tend to a limit $p_{t}(x, y)$ which does not depend on the choice of $D_{n}$ (this follows from [19, Ch. 1]). There exists a continuous Markov process $\xi$ in $E$ with the transition function $p_{t}(x, d y)=p_{t}(x, y) d y$ (see, e.g., [6]). We call it an L-diffusion in $E$.

1.3. $G$-equation. Markov semigroup, Green's function $g$ and Green's operator $G$ for an $L$-diffusion $\xi$ are defined by the formulae

$$
\begin{gathered}
T_{t} f(x)=\int_{E} p_{t}(x, d y) f(y), \\
g(x, y)=\int_{0}^{\infty} p_{t}(x, y) d t
\end{gathered}
$$

and

$$
G f(x)=\int_{0}^{\infty} T_{t} f(x) d t=\int_{E} g(x, y) f(y) d y
$$


A positive Borel function $h$ is called excessive if, for all $x \in E, T_{t} h(x) \leq h(x)$ and $T_{t} h(x) \rightarrow h(x)$ as $t \rightarrow 0$. The case $h(x)=\infty$ for all $x$ is excluded. Since $p_{t}(x, y)>0$, the set $\{x: h(x)=\infty\}$ has the Lebesgue measure 0 . There exist only two possibilities: either $g(x, y)=\infty$ for all $x, y \in E$ or $g(x, y)<\infty$ for $x \neq y$. In the first case, constants are the only excessive functions and all problems treated in this paper are trivial. Therefore we concentrate on the second case.

Let $1<\alpha \leq 2$. One of our goals is to find for which excessive functions $h$ the equation

$$
v+G\left(v^{\alpha}\right)=h
$$

(we call it $G$-equation) has a solution. ${ }^{3}$ Note that if (1.14) holds almost everywhere, then

$$
\tilde{v}= \begin{cases}h-G\left(v^{\alpha}\right) & \text { on }\{h<\infty\} \\ \infty & \text { on }\{h=\infty\}\end{cases}
$$

satisfies (1.14) everywhere.

Fix an arbitrary point $c \in E$ and put

$$
\begin{aligned}
k(x, y) & =\frac{g(x, y)}{g(c, y)} \quad \text { if } y \neq c, \\
& =0 \quad \text { otherwise. }
\end{aligned}
$$

There exist [see, e.g., [7]] a continuous injective mapping from $E$ to a compact metrizable space $\hat{E}$ and an extension of $k(x, y)$ to $E \times \hat{E}$ such that:

1.3.A. For every $x \in E, k(x, y) \rightarrow k(x, z)$ as $y \rightarrow z \in \hat{E} \backslash E$.

1.3.B. If $k\left(\cdot, y_{1}\right)=k\left(\cdot, y_{2}\right)$, then $y_{1}=y_{2}$.

We call $\hat{E}$ the Martin space. The set $\partial E=\hat{E} \backslash E$ is called the Martin boundary. For every $y \in E, h(x)=g(x, y)$ is an extremal excessive function. ${ }^{4}$ We denote by $E^{*}$ the set of all $y \in \partial E$ such that $h(x)=k(x, y)$ is an extremal excessive function. ( $E^{*}$ is a Borel subset of $\partial E$.) Every excessive function $h$ has a unique representation

$$
h=G \eta+K \nu
$$

where $\eta$ is a $\sigma$-finite measure on $E, \nu$ is a finite measure on $E^{*}$ and

$$
G \eta(x)=\int_{E} g(x, y) \eta(d y), \quad K \nu(x)=\int_{E^{*}} k(x, y) \nu(d y)
$$

(cf. (1.6)). Note that $\eta(\Gamma)<\infty$ for every compact $\Gamma \subset E$. Indeed, if $h\left(x_{0}\right)<\infty$, then $a \eta(\Gamma) \leq G \eta\left(x_{0}\right)<\infty$ where $a=\inf _{y \in \Gamma} g\left(x_{0}, y\right)>0$.

Function $f=K \nu$ is $L$-harmonic, that is it satisfies equation $L f=0$. $L$-harmonic functions can be also characterized by the following mean value property: for every bounded open set $D$ such that $\bar{D} \subset E$,

$$
\Pi_{x} f\left(\xi_{\tau}\right)=f(x) \quad \text { for all } x \in E
$$

where $\tau$ is the first exit time from $D$.

\footnotetext{
${ }^{3}$ (Cf. (1.3).) When speaking about solutions of $G$-equation, we always mean positive solutions.

${ }^{4}$ This means if $h=h_{1}+h_{2}$ and if $h_{1}, h_{2}$ are excessive, then $h_{1}, h_{2}$ are proportional to $h$.
} 
We fix $\alpha \in(1,2]$. Green's capacity $C G$ is defined on compact subsets of $E$ by the formula

$$
C G(\Gamma)=\sup \left\{\eta(\Gamma): \int_{E} g(c, x) d x\left[\int_{\Gamma} g(x, y) \eta(d y)\right]^{\alpha} \leq 1\right\} .
$$

Analogously, the Martin capacity $C K$ is defined on compact subsets of $\partial E$ by the formula

$$
C K(\Gamma)=\sup \left\{\nu(\Gamma): \int_{E} g(c, x) d x\left[\int_{\Gamma} k(x, y) \nu(d y)\right]^{\alpha} \leq 1\right\} .
$$

[By a Choquet theorem [3], $C G$ and $C K$ can be extended to all analytic subsets of $E$ and $E^{*}$.] If $\eta$ is a measure on $E$, then writing $\eta \prec C G$ means that $\eta(\Gamma)=0$ if $C G(\Gamma)=0$. Writing $\nu \prec C K$ has an analogous meaning.

It follows from the results in Sections 2 and 3 that:

Theorem 1.1. If $h=G \eta+K \nu$ and if

$$
\eta \prec C G, \quad \nu \prec C K,
$$

then $G$-equation (1.14) has a solution $v$ which is defined uniquely on the set $E(h)=$ $\{h<\infty\}$.

1.4. Operators $\mathcal{G}$ and $\mathcal{K}$. Let $\xi$ be an $L$-diffusion in a bounded smooth domain $D$ stopped at the first exit time $\tau$ from $D$. We introduce operators $\mathcal{G}$ and $\mathcal{K}$ acting on functions with the domain $S=\mathbb{R}_{+} \times E$ by the formulae

$$
\mathcal{G} f(t, x)=\int_{0}^{t} d s \int_{D} p_{s}(x, d y) f(t-s, y)=\Pi_{x} \int_{0}^{\tau \wedge t} f\left(t-s, \xi_{s}\right) d s,
$$

and $^{5}$

$$
\mathcal{K} f(t, x)=\Pi_{x} f\left(t-\tau, \xi_{\tau}\right) .
$$

If $f(t, x)=f(x)$ does not depend on $t$, then

$$
\begin{aligned}
& \mathcal{G} f(t, x)=\Pi_{x} \int_{0}^{t} f\left(\xi_{s}\right) d s \rightarrow G f(x), \\
& \mathcal{K} f(t, x)=\Pi_{x} f\left(\xi_{\tau}\right) 1_{\tau \leq t} \rightarrow K f(x)
\end{aligned}
$$

as $t \rightarrow \infty$. Here $G$ is defined by (1.13) and ${ }^{6}$

$$
K f(x)=\Pi_{x} f\left(\xi_{\tau}\right) .
$$

\footnotetext{
${ }^{5}$ We extend each function to $\mathbb{R} \times E$ by setting it equal to zero for negative $t$.

${ }^{6}$ Operator (1.26) is a particular case of the operator $K$ defined by (1.18): if $E=D$ is a bounded smooth domain, then $E^{*}=\partial D$ and $\Pi_{x} f\left(\xi_{\tau}\right)=\int_{\partial D} k(x, y) \nu(d y)$ for $\nu(d y)=f(y) a(d y)$ where $k$ is the Poisson kernel and $a$ is the surface area on $\partial D$. Writing the same letter for both operators should cause no confusion since one operator is applied only in the context of a smooth domain $D$ and the second one only in the context of the Martin boundary of $E$.
} 
The boundary of a cylinder $Q=\mathbb{R}_{+} \times D$ consists of the side surface $A=$ $(0, \infty) \times \partial D$ and the bottom $B=\{0\} \times \bar{D}$. Besides the boundary value problem (1.2), we consider also a boundary value problem for a parabolic equation

$$
\begin{aligned}
\frac{\partial u}{\partial t}-L u+u^{\alpha} & =\rho & & \text { in } Q, \\
u & =\sigma & & \text { on } A, \\
u & =0 & & \text { on } B .
\end{aligned}
$$

If $\rho$ and $\sigma$ are Hölder continuous, then (1.27) is equivalent to the integral equation

$$
u+\mathcal{G}\left(u^{\alpha}\right)=\mathcal{G} \rho+\mathcal{K} \sigma .
$$

1.5. Superdiffusions. Let $\xi=\left(\xi_{t}, \Pi_{x}\right)$ be a Markov process in a measurable space $(E, \mathcal{B})$ and let $\mathcal{M}=\mathcal{M}(E)$ be the space of all finite measures on $\mathcal{B}$. A $(\xi, \alpha)$ superprocess is a Markov process $X=\left(X_{t}, P_{\mu}\right)$ in $\mathcal{M}$ which satisfies the condition: for every $\mu \in \mathcal{M}$ and every positive $\mathcal{B}$-measurable function $f$,

$$
\begin{aligned}
P_{\mu} \exp \left\langle-f, X_{t}\right\rangle & =\exp \left\langle-u_{t}, \mu\right\rangle, \\
u_{t}(x)+\Pi_{x} \int_{0}^{t} u_{t-s}\left(\xi_{s}\right)^{\alpha} d s & =\Pi_{x} f\left(\xi_{t}\right) .
\end{aligned}
$$

We say that $X$ is an $(L, \alpha)$-superdiffusion if $X$ is a right process and $\xi$ is an $L$ diffusion. The existence of such processes for $1<\alpha \leq 2$ is proved, for instance, in [13] (we refer to [8] and [9] for the history of this subject starting from the pioneering work of Watanabe and Dawson).

In the theory of diffusion, a fundamental role is played by random points $\xi_{\tau}$ corresponding to the first exit times from open sets $D$. An analogous role in the theory of superdiffusion is played by exit measures $X_{D}$. In contrast to $\xi_{\tau}$ which can be defined through $\xi_{t}$, it is impossible, in general, to define $X_{D}$ in terms of $X_{t}$. The probability distribution of $X_{D}$ is defined by formulae similar to (1.29):

$$
\begin{aligned}
P_{\mu} \exp \left\langle-f, X_{D}\right\rangle & =\exp \langle-u, \mu\rangle, \\
u(x)+\Pi_{x} \int_{0}^{\tau} u\left(\xi_{s}\right)^{\alpha} d s & =\Pi_{x} f\left(\xi_{\tau}\right) .
\end{aligned}
$$

The joint probability distribution of $X_{t_{1}}, \ldots, X_{t_{n}}$ is determined by (1.29) and the Markov property of $X$. Analogously, the joint probability distribution of $X_{D_{1}}, \ldots, X_{D_{n}}$ can be evaluated by using (1.30) and the following Markov property: for every positive $\mathcal{F}_{\supset D}$-measurable $Y$,

$$
P_{\mu}\left\{Y \mid \mathcal{F}_{\subset D}\right\}=P_{X_{D}} Y
$$

where $\mathcal{F}_{\subset D}$ is the $\sigma$-algebra generated by $X_{D^{\prime}}$ with $D^{\prime} \subset D$ and $\mathcal{F}_{\supset D}$ the $\sigma$-algebra generated by $X_{D^{\prime \prime}}$ with $D^{\prime \prime} \supset D$.

We need even a wider class of exit measures [for instance, measures corresponding to the exit from $D$ before time $t]$. We introduce a random measure $\left(X_{Q}, P_{\mu}\right)$ for 
an arbitrary open set $Q$ in $S=\mathbb{R}_{+} \times E$ and an arbitrary finite measure $\mu$ on the Borel $\sigma$-algebra in $S$. Its probability distribution is defined by the formulae

$$
\begin{aligned}
& P_{\mu} \exp \left\langle-f, X_{Q}\right\rangle=\exp \langle-u, \mu\rangle, \\
& u(r, x)+\Pi_{r, x} \int_{r}^{\tau^{r}} u\left(s, \xi_{s}\right)^{\alpha} d s=\Pi_{r, x} f\left(\tau^{r}, \xi_{\tau^{r}}\right)
\end{aligned}
$$

where

$$
\tau^{r}=\inf \left\{t: t \geq r,\left(t, \xi_{t}\right) \notin Q\right\}
$$

is the first, after $r$, exit time of $\xi$ from $Q$ and $\Pi_{r, x} Y=\Pi_{x} \theta_{-r} Y$ describes a Markov process with transition function $p_{t}(x, d y)$ which starts at time $r$ from point $x$. The joint probability distribution of $X_{Q_{1}}, \ldots, X_{Q_{n}}$ is determined by (1.33) and by the property: for every positive $\mathcal{F}_{\supset Q^{-}}$-measurable $Y$,

$$
P_{\mu}\left\{Y \mid \mathcal{F}_{\subset Q}\right\}=P_{X_{Q}} Y
$$

where $\mathcal{F}_{\subset Q}$ is the $\sigma$-algebra generated by $X_{Q^{\prime}}$ with $Q^{\prime} \subset Q$ and $\mathcal{F}_{\supset Q}$ the $\sigma$-algebra generated by $X_{Q^{\prime \prime}}$ with $Q^{\prime \prime} \supset Q$.

The existence of a family $\left(X_{Q}, P_{\mu}\right)$ subject to conditions (1.32) and (1.34) is proved in [8].

Formula $j_{r}(x)=(r, x)$ defines a mapping from $E$ to $S$. If $\mu$ is a measure on $E$, then $j_{r}(\mu)$ is a measure on $S$ concentrated on $\{r\} \times E$. We set $P_{j_{r}(\mu)}=P_{r, \mu}$. It follows from (1.32) that

$$
\begin{aligned}
P_{r, \mu} \exp \left\langle-f, X_{Q}\right\rangle & =\exp \left\{-\int_{E} u(r, x) \mu(d x)\right\}, \\
u(r, x)+\Pi_{x} \int_{0}^{\tau} u\left(s+r, \xi_{s}\right)^{\alpha} d s & =\Pi_{x} f\left(\tau+r, \xi_{\tau}\right) .
\end{aligned}
$$

Formulae (1.29) and (1.30) can be considered as special cases of (1.35) if we identify $X_{t}$ and $X_{D}$ with the exit measures from $S_{<t}=[0, t) \times E$ and from $\mathbb{R}_{+} \times D$, projected on $E$.

If $\tau$ is the first exit time from $D$, then $\tau(t)=\tau \wedge t$ is the first exit time from $Q_{t}=$ $[0, t) \times D$. We call the process $\tilde{X}_{t}=X_{Q_{t}}$ an $(L, \alpha)$-superdiffusion stopped at the exit from $D$. If $f(t, x)=f(x)$ vanishes outside $D$ and if $v_{t}(x)=-\log P_{x} \exp \left\langle-f, \tilde{X}_{t}\right\rangle$, then $v_{t-r}(x)=-\log P_{r, x} \exp \left\langle-f, X_{Q_{t}}\right\rangle$ and (1.35) implies

$$
\begin{aligned}
P_{\mu} \exp \left\langle-f, \tilde{X}_{t}\right\rangle & =\exp \left\langle-v_{t}, \mu\right\rangle, \\
v_{t}(x)+\Pi_{x} \int_{0}^{\tau(t)} v_{t-s}\left(\xi_{s}\right)^{\alpha} d s & =\Pi_{x} f\left(\xi_{\tau(t)}\right) .
\end{aligned}
$$

Formula (1.36) can be obtained from (1.29) by replacing $\xi$ with an $L$-diffusion stopped at the exit from $D$.

The shift operators $\theta_{t}$ of a time-homogeneous process $\xi$ induce analogous operators for $X$ (see [14, Section 1.12]). We have $X_{s}\left(\theta_{t} \omega\right)=X_{s+t}(\omega)$ and, if $Q=\mathbb{R}_{+} \times D$, then $X_{Q}\left(\theta_{t} \omega, \Gamma\right)=X_{Q_{t}}(\omega, \Gamma+t)$ where $Q_{t}=S_{<t} \cup\left\{\gamma_{t}(Q)\right\}$ with $\gamma_{t}(r, x)=(r+t, x)$. 
It follows from (1.35) that

$$
P_{\mu} \int_{Q} f(s, x) X_{Q}(d s, d x)=\int \mu(d x) \Pi_{x} f\left(\tau, \xi_{\tau}\right)
$$

[it is sufficient to apply (1.35) to $\lambda f$ and to take the derivative with respect to $\lambda$ at $\lambda=0]$.

The following result (see Theorem I.1.8 in [8]) provides a link between superprocesses and the $G$-equation.

Theorem A. Suppose that $\tilde{X}$ is an $(L, \alpha)$-superdiffusion stopped at the exit from $D, \rho$ is a positive Borel function on $\bar{D}$ vanishing on $\partial D$ and $\sigma$ is a positive Borel function on $\partial D$. Then

$$
v(x)=-\log P_{x} \exp \left\{-\left[\int_{0}^{\infty}\left\langle\rho, \tilde{X}_{t}\right\rangle d t+\left\langle\sigma, X_{D}\right\rangle\right]\right\}
$$

is a solution of the G-equation (1.14) where $G$ is Green's operator for L-diffusion in $D, K$ is given by (1.26) and ${ }^{7}$

$$
h=G \rho+K \sigma .
$$

Moreover, for every $\mu \in \mathcal{M}(D)$,

$$
P_{\mu} \exp \left\{-\left[\int_{0}^{\infty}\left\langle\rho, \tilde{X}_{t}\right\rangle d t+\left\langle\sigma, X_{D}\right\rangle\right]\right\}=e^{-\langle v, \mu\rangle} .
$$
[16]].

We also need another implication of Theorem I.1.8 in [8] [cf. Theorem 1.1 in

Theorem B. Let $\tilde{X}, D$ and $\rho$ be the same as in Theorem A and let $\sigma$ be a positive Borel function on $\bar{D}$ vanishing on $D$. Then

$$
u(t, x)=-\log P_{x} \exp \left\{-\left[\int_{0}^{t}\left\langle\rho, \tilde{X}_{s}\right\rangle d s+\left\langle\sigma, \tilde{X}_{t}\right\rangle\right]\right\}
$$

is a solution of the equation (1.28). Moreover, for every $\mu \in \mathcal{M}(D),{ }^{8}$

$$
P_{\mu} \exp \left\{-\left[\int_{0}^{t}\left\langle\rho, \tilde{X}_{s}\right\rangle d s+\left\langle\sigma, \tilde{X}_{t}\right\rangle\right]\right\}=\exp \left\langle-u^{t}, \mu\right\rangle .
$$

The range $\mathcal{R}$ of a superprocess $X$ is the smallest closed subset of $E$ which supports all measures $X_{t}$ (it supports, a.s., every exit measure $X_{D}$ ). We denote by $\mathcal{R}^{*}$ the minimal closed subset of the Martin space $\hat{E}$ which supports all measures $X_{t}$. A set $\Gamma \subset E$ is called $\mathcal{R}$-polar if $P_{x}\{\mathcal{R} \cap \Gamma \neq \emptyset\}=0$ for all $x \notin \Gamma$. A subset $\Gamma$ of the Martin boundary $\partial E$ is called $\mathcal{R}^{*}$-polar if $P_{x}\left\{\mathcal{R}^{*} \cap \Gamma \neq \emptyset\right\}=0$ for all $x \in E .^{9}$

We prove in Section 4:

\footnotetext{
${ }^{7}$ For $x \in \partial D, u(x)=h(x)=\sigma(x)$.

${ }^{8}$ We set $u^{t}(x)=u(t, x)$.

${ }^{9} \mathcal{R}^{*}$-polarity is introduced only on $\partial E$ because $\mathcal{R}^{*} \cap \Gamma=\mathcal{R} \cap \Gamma$ for every compact $\Gamma \subset E$.
} 
Theorem 1.2. If $h=G \eta+K \nu$ and if the G-equation (1.14) has a solution, then $\eta$ does not charge $\mathcal{R}$-polar sets and $\nu$ does not charge $\mathcal{R}^{*}$-polar sets.

We say that $\Gamma \subset E$ is $G$-polar if $C G(\Gamma)=0$ and that $\Gamma \subset \partial E$ is $K$-polar if $C K(\Gamma)=0$. By Theorem 1.1 in [17] [cf. Theorem 1.6 in [12]], the classes $\mathcal{R}$-polar and $G$-polar sets coincide. Theorems 1.1 and 1.2 imply:

1.5.A. All $\mathcal{R}^{*}$-polar sets are $K$-polar.

Indeed, if $\Gamma$ is compact and if $C K(\Gamma)>0$, then by (1.21), there exists a measure $\nu \neq 0$ concentrated on $\Gamma$ such that

$$
\int_{E} g(c, x) d x\left[\int k(x, y) \nu(d y)\right]^{\alpha}<\infty
$$

which implies

$$
\int_{E} g(c, x) d x\left[\int_{B} k(x, y) \nu(d y)\right]^{\alpha}<\infty
$$

for every $B$. Hence $\nu(B)=0$ for all $K$-polar sets $B$. By Theorems 1.1 and 1.2, $\nu(B)=0$ for all $\mathcal{R}^{*}$-polar sets $B$. Therefore $\Gamma$ is not $\mathcal{R}^{*}$-polar.

If $\xi$ is an $L$-diffusion in a bounded smooth domain of $\mathbb{R}^{d}$, then a stronger result than 1.5.A follows from Theorem 1.2 in [17]:

1.5.B. The classes of $\mathcal{R}^{*}$-polar and $K$-polar sets coincide.

It remains an open problem if 1.5.B holds in the general case. If it holds for a diffusion $\xi$ and if $X$ is the corresponding superdiffusion, then each of Theorems 1.1-1.2 gives necessary and sufficient conditions on $h$ for the existence of a solution of (1.14).

1.6. Additive functionals. Let $X$ be a superdiffusion. We denote by $\mathcal{F}_{t}$ the $\sigma$-algebra in $\Omega$ generated by the exit measures $X_{Q}$ for all $Q \subset S_{<t}$. A function $A_{t}(\omega)$ from $[0, \infty] \times \Omega$ to $[0, \infty]$ is called an additive functional of $X$ if:

1.6.A. For every $\omega, A_{t}$ is monotone increasing in $t$.

1.6.B. $A_{t}$ is measurable with respect to the completion of $\mathcal{F}_{t}$ with respect to all measures $P_{\mu}, \mu \in \mathcal{M}(E)$.

1.6.C. For every $\omega, A_{t}$ is left continuous in $t$.

1.6.D. $A_{s+t}=A_{s}+\theta_{s} A_{t}$ for all pairs $s, t$ and all $\omega .^{10}$

All these conditions hold for

$$
A_{t}=\int_{0}^{t}\left\langle\rho, X_{s}\right\rangle d s
$$

where $\rho$ is an arbitrary positive Borel function. By a limit procedure, we construct, starting from (1.43), a class of functionals for which a weaker form of condition 1.6.D holds.

We say that a set $\Lambda$ is $\xi$-polar if $\Pi_{x}\left\{\xi_{t} \notin \Lambda\right.$ for all $\left.t>0\right\}=1$ for all $x$. All $\xi$-polar sets have the Lebesgue measure 0 . A subset $\mathcal{N}$ of $\mathcal{M}(E)$ is called exceptional if the set $\left\{x: \delta_{x} \in \mathcal{N}\right\}$ is $\xi$-polar and if, for all stopped superdiffusions $\tilde{X}$ and for every $\mu \notin \mathcal{N}, P_{\mu}\left\{\tilde{X}_{t} \notin \mathcal{N}\right.$ for all $\left.t\right\}=1$.

\footnotetext{
${ }^{10}$ Let $\beta(\omega)=\sup \left\{t: A_{t}(\omega)<\infty\right\}$. Then there exists a unique measure $A(\omega, d t)$ on $[0, \beta(\omega))$ such that $A[0, t)=A_{t}$ for all $t<\beta$.
} 
If $h$ is an arbitrary excessive function, then the set $\Lambda(h)=\{x: h(x)=\infty\}$ is $\xi$-polar and the set $\mathcal{N}(h)=\{\mu:\langle h, \mu\rangle=\infty\}$ is exceptional.

We say that $A$ is an additive functional with an exceptional set $\mathcal{N}$ if $A$ satisfies 1.6.A, B, C and:

1.6.D*. $A_{s+t}=A_{s}+\theta_{s} A_{t}$ for all $s, t, \omega \in \Omega_{0}$ and $P_{\mu}\left(\Omega_{0}\right)=1$ for all $\mu \notin \mathcal{N}$.

Two additive functionals $A$ and $\tilde{A}$ are called equivalent if there exists an exceptional set $\mathcal{N}$ such that $P_{\mu}\left\{A_{t}=\tilde{A}_{t}\right.$ for all $\left.t\right\}=1$ for all $\mu \notin \mathcal{N}$.

Let $h$ be an excessive function. An additive functional $A$ with an exceptional set $\mathcal{N}$ is called a linear additive functional with potential $h$ if, for every $\mu \notin \mathcal{N}$,

$$
P_{\mu} A_{\infty}=\langle h, \mu\rangle .
$$

If $G \rho(x)<\infty$ for some $x$, then the additive functional (1.43) is linear with potential $G \rho$ (condition (1.44) holds for every $\mu$ ).

Theorem 1.3. If $h=G \eta+K \nu$ and if $\eta \prec C G, \quad \nu \prec C K$, then $h$ is the potential of a linear additive functional $A$ of $X$ with an exceptional set $\mathcal{N}$. For every $\mu \notin \mathcal{N}$,

$$
P_{\mu} e^{-A_{\infty}}=e^{-\langle v, \mu\rangle}
$$

where $v$ is a solution of the G-equation (1.14).

Theorem 1.3 is proved in Section 3. Theorem 1.1 follows immediately from Theorem 1.3 and a uniqueness Theorem 2.1.

Remark. The construction of $A$ in Section 3 implies that $A$ depends linearly on $h$. More precisely, if $A^{i}$ corresponds to $h^{i}$, then, for every $c_{1}, c_{2} \geq 0$, the functional $A$ corresponding to $c_{1} h^{1}+c_{2} h^{2}$ is equivalent to $c_{1} A^{1}+c_{2} A^{2}$. Therefore, if $h, \tilde{h}$ and $h-\tilde{h}$ are excessive functions and if $v, \tilde{v}$ are the solutions of (1.14) corresponding to $h$ and $\tilde{h}$, then $\tilde{v} \leq v$ outside a $\xi$-polar set.

In Section 4 we establish:

Theorem 1.4. If $h$ is the potential of a linear additive functional with an exceptional set $\mathcal{N}$, then $h=G \eta+K \nu$ with $\eta$ vanishing on all $\mathcal{R}$-polar sets and $\nu$ vanishing on all $\mathcal{R}^{*}$-polar sets.

Linear additive functionals of superprocesses have been introduced in [11] (in a time-inhomogeneous setting). There a linear additive functional corresponding to a bounded excessive function $h$ was constructed for a $(\xi, 2)$-superprocess where $\xi$ is an arbitrary right Markov process. (No exceptional set is needed in this case.)

The case of an $(L, \alpha)$-superdiffusion with an arbitrary $\alpha \in(1,2]$ was investigated in [15]. For $h=G \eta$ with $\eta \prec C G$, a functional $A$ was constructed, subject to conditions 1.6.A, B with the property, for every $\mu \in \mathcal{M}^{0}$,

1.6.D**. $A_{s+t}=A_{s}+\theta_{s} A_{t} P_{\mu}$-a.s. for all $s, t$.

Here $\mathcal{M}^{0}$ is the set of measures of the form $\mu(d x)=\rho(x) d x$ with $\int \rho(x)^{\alpha^{\prime}} d x<\infty$ where $\alpha^{\prime}=\alpha /(\alpha-1)$. Condition (1.44) was proved also only for $\mu \in \mathcal{M}^{0}$. (Note that $\mathcal{M}^{0}$ is not the complement of an exceptional set!)

Recent results of Le Gall [23] on additive functionals of the Brownian snake can be translated into our language as follows: if $h=K \nu$ with $\nu \prec C K$, then there exists a functional of an $(\Delta, 2)$-superdiffusion which satisfies conditions 1.6.A, B, $\mathrm{C},(1.44)$ and 1.6. $\mathrm{D}^{* *}$ for $P_{x}$ for almost all $x$. 
Additive functionals with an exceptional set have been introduced, in a different context, by Fukushima [20]. In his setting, $X$ is a symmetric Markov process associated with a Dirichlet form and an exceptional set is a polar subset of the state space (in the sense of theory of Dirichlet spaces).

1.7. We have the following logical implications: $\mathcal{A} \Longrightarrow \mathcal{B} \Longrightarrow \mathcal{C} \Longrightarrow \mathcal{D}$ where:

$\mathcal{A}: h=G \eta+K \nu$ with $\eta \prec C G, \nu \prec C K$;

$\mathcal{B}: h$ is the potential of a linear additive functional $A$ with an exceptional set $\mathcal{N}$. Moreover for every $\mu \notin \mathcal{N}$,

$$
P_{\mu} e^{-A_{\infty}}=e^{-\langle v, \mu\rangle}
$$

where $v$ is a solution of the $G$-equation (1.14).

$\mathcal{C}: h$ is the potential of a linear additive functional $A$.

$\mathcal{D}: h=G \eta+K \nu$ with $\eta$ vanishing on all $\mathcal{R}$-polar sets and $\nu$ vanishing on all $\mathcal{R}^{*}$-polar sets.

We get $\mathcal{A} \Longrightarrow \mathcal{B}$ by Theorem 1.3 and $\mathcal{C} \Longrightarrow \mathcal{D}$ by Theorem 1.4 . The implication $\mathcal{B} \Longrightarrow \mathcal{C}$ is trivial.

If 1.5.B holds for a diffusion $\xi$ and if $X$ is the corresponding superdiffusion, then $\mathcal{D} \Longrightarrow \mathcal{A}$ and all four statements $\mathcal{A}, \mathcal{B}, \mathcal{C}$ and $\mathcal{D}$ are equivalent. In particular, this is true if $\xi$ is an $L$-diffusion in a bounded smooth domain $D$. This also is true for an arbitrary domain $E$ if we consider only excessive functions $h=G \eta$ (in other words if we set $\nu=0$ ).

Acknowledgments. The authors are greatly indebted to N. V. Krylov. The first author, during his visit to the University of Minnesota in January 1995, had a number of stimulating conversations with Krylov on the subject of quasi-linear PDEs. A result of these discussions was a considerable simplification of proofs in Theorems 2.1 and 2.2 .

\section{2. $G$-EQUATION}

\subsection{Monotonicity and uniqueness.}

Theorem 2.1. Let $\xi$ be an L-diffusion stopped at the first exit time $\tau$ from an open set $D$, and let $G, K$ be given by (1.13),(1.26). Suppose that $\eta$ is a measure on $D$, $u, \hat{u}, \sigma \geq 0$ and, for almost all $x$,

$$
\hat{u}+G\left(\hat{u}^{\alpha}\right)=u+G\left(u^{\alpha}\right)+G \eta+K \sigma<\infty .
$$

Then $\hat{u} \geq u$ at every point of the set (2.1). If $\eta=0$ and $\sigma=0$, then $\hat{u}=u$ on the same set.

An analogous result holds for the $\mathcal{G}$-equation. For every measure $\eta$ on $S$ we put

$$
\mathcal{G} \eta(t, x)=\int_{0}^{t} \int_{D} p_{t-s}(x, y) \eta(d s, d y)
$$

(cf. (1.23)). If $\eta(d s, d y)=d s \eta(d y)$, then

$$
\mathcal{G} \eta(t, x)=\int_{0}^{t} d s \int_{D} p_{s}(x, y) \eta(d y) \rightarrow G \eta(x)
$$

as $t \rightarrow \infty$. 
Theorem 2.1*. Let $\xi$ be the same as in Theorem 2.1 and let $\mathcal{G}, \mathcal{K}$ be given by (2.2),(1.24). Suppose that $\eta$ is a measure on $S, u, \hat{u}, \sigma \geq 0$ and, for almost all $t, x$,

$$
\hat{u}+\mathcal{G}\left(\hat{u}^{\alpha}\right)=u+\mathcal{G}\left(u^{\alpha}\right)+\mathcal{G} \eta+\mathcal{K} \sigma<\infty .
$$

Then $\hat{u} \geq u$ at every point of the set (2.4). If $\eta=0$ and $\sigma=0$, then $\hat{u}=u$ on the same set.

We use as a tool a process $\left(\xi_{s}, \Pi_{x}^{t, y}\right)$ with $x, y \in D$. Its finite-dimensional distributions are given by the formula

$$
\begin{gathered}
\prod_{x}^{t, y}\left\{\xi_{t_{1}} \in d y_{1}, \ldots, \xi_{t_{n}} \in d y_{n}, t_{n}<t<\tau\right\} \\
=p_{t_{1}}\left(x, d y_{1}\right) p_{t_{2}-t_{1}}\left(y_{1}, d y_{2}\right) \ldots p_{t_{n}-t_{n-1}}\left(y_{n-1}, d y_{n}\right) p_{t-t_{n-1}}\left(y_{n}, y\right)
\end{gathered}
$$

for all $0<t_{1}<\cdots<t_{n}<t$. (Here $p_{t}(x, d y)$ is the transition function and $p_{t}(x, y)$ is the transition density of the part of $\xi$ in $D$. $)^{11}$

Let $f$ be a positive Borel function. Formula

$$
p_{t}^{\varphi}(x, y)=\Pi_{x}^{t, y}\left\{\exp \left\{-\int_{0}^{t} \varphi\left(\xi_{s}\right) d s\right\}\right\}
$$

defines the transition density of a Markov process obtained from $\xi$ by killing with rate $f(x)$ at point $x$.

Operator $G_{\varphi}$ corresponding to $p^{\varphi}$ by $(2.2)$ can be expressed by formula

$$
\mathcal{G}_{\varphi} \rho(t, x)=\Pi_{x} \int_{0}^{\tau \wedge t} d s \rho\left(t-s, \xi_{s}\right) \exp \left\{-\int_{0}^{s} \varphi\left(t-r, \xi_{r}\right) d r\right\} .
$$

We prove Theorem 2.1*. (Proof of Theorem 2.1 is similar but simpler.) We need two lemmas.

Lemma 2.1. ${ }^{12}$ (i) Let $\rho$ be a Borel function on $S$. Equation

$$
\mathcal{G} \rho-\mathcal{G}_{\varphi} \rho=\mathcal{G}_{\varphi}(\varphi \mathcal{G} \rho)
$$

holds on the set $\{\mathcal{G}|\rho|<\infty\}$.

(ii) If $\eta$ is a measure on $S$, then

$$
\mathcal{G} \eta-\mathcal{G}_{\varphi} \eta=\mathcal{G}_{\varphi}(\varphi \mathcal{G} \eta)
$$

on the set $\{\mathcal{G} \eta<\infty\}$.

(iii) For every positive Borel $\sigma$, equation

$$
\mathcal{K} \sigma-\mathcal{K}_{\varphi} \sigma=\mathcal{G}_{\varphi}(\varphi \mathcal{K} \sigma)
$$

holds on the set $\{\mathcal{K} \sigma<\infty\}$.

\footnotetext{
${ }^{11}$ Normalized measure $\Pi_{x}^{t, y}$ can be obtained by conditioning the diffusion $\xi$ started from point $x$ to come at point $y$ at time $t$.

${ }^{12}$ Cf. [5]. This result can be interpreted as the resolvent form of the Feynman-Kac formula.
} 
Proof. $1^{\circ}$. It is sufficient to check (2.8) for $\rho \geq 0$. We use (1.23) and (2.7), the Markov property of $\xi$, Fubini's theorem and relation

$$
\int_{0}^{s} d a Y_{a} \exp \left\{-\int_{0}^{a} Y_{r} d r\right\}=1-\exp \left\{-\int_{0}^{s} Y_{r} d r\right\}
$$

which we apply to $Y_{s}=\varphi\left(t-s, \xi_{s}\right)$.

$2^{\circ}$. Put

$$
\rho_{\varepsilon}(x)=\int_{D} p_{\varepsilon}(x, y) \eta(d y) .
$$

Note that

$$
G \rho_{\varepsilon}(x)=\int_{\varepsilon}^{\infty} d t \int_{D} p_{t}(x, y) \eta(d y)
$$

We get (2.9) by applying (2.8) to $\rho_{\varepsilon}$ and by passing to the limit as $\varepsilon \rightarrow 0$.

$3^{\circ}$. Formula (2.10) can be proved in the same way as $(2.8)$.

Lemma 2.2. Suppose that $\varphi, \sigma \geq 0$ and that, for almost all $t, x$,

$$
\mathcal{G} \eta+\mathcal{K} \sigma+\mathcal{G}|\varphi w|<\infty
$$

and

$$
w+\mathcal{G}(\varphi w)=\mathcal{G} \eta+\mathcal{K} \sigma .
$$

Then

$$
w=\mathcal{G}_{\varphi} \eta+\mathcal{K}_{\varphi} \sigma
$$

at every point $(t, x)$ where (2.13) and (2.14) hold.

Proof. We have

$$
\mathcal{G}_{\varphi}(\varphi w)+\mathcal{G}_{\varphi}[\varphi \mathcal{G}(\varphi w)]=\mathcal{G}_{\varphi}(\varphi \mathcal{G} \eta)+\mathcal{G}_{\varphi}(\varphi \mathcal{K} \sigma)
$$

On the set defined by (2.13) and (2.14), the left side in (2.16) is equal to $\mathcal{G}(\varphi w)$ by (2.8) and, the right side is equal to $\mathcal{G} \eta+\mathcal{K} \sigma-\mathcal{G}_{\varphi} \eta-\mathcal{K}_{\varphi} \sigma$ by (2.8) and (2.10). Therefore $\mathcal{G}(\varphi w)=\mathcal{G} \eta+\mathcal{K} \sigma-\mathcal{G}_{\varphi} \eta-\mathcal{K}_{\varphi} \sigma$ and (2.15) follows from (2.14).

Proof of Theorem 2.1*. Denote by $\hat{S}$ the set defined by (2.4). Put $w=\hat{u}-u$ on $\hat{S}$ and $w=0$ on $E \backslash \hat{S}$. There exists a function $\varphi \geq 0$ such that $\hat{u}^{\alpha}-u^{\alpha}=\varphi w$ a.e. Equation (2.4) implies (2.14). Since $G|\varphi w| \leq G\left(u^{\alpha}\right)+G\left(\hat{u}^{\alpha}\right)<\infty$ on $\hat{S}$, Theorem $2.1^{*}$ follows from Lemma 2.2 . 
2.2. Properties of $G$ and $\mathcal{G}$. In this subsection we deal with operators corresponding to an $L$-diffusion $\xi$ in a bounded smooth domain $D$. We denote by $\|u\|$ the norm of $u$ in $L^{1}(D)$. For a function $f$ on $S=\mathbb{R}_{+} \times D$ and for $b \in \mathbb{R}_{+}$, we set

$$
\ell_{b}(f)=\int_{0}^{b} \int_{D}|f(r, x)| d r d x .
$$

We need the following results.

2.2.A. There is a constant $C$ such that

$$
\int_{D} g(x, y) d x \leq C \quad \text { for all } y \in D .
$$

2.2.A*. For every $b>0$, there exists a constant $C$ such that

$$
\int_{D} p_{t}(x, y) d x \leq C \quad \text { for all } y \in D, 0<t \leq b .
$$

2.2.B. If $f_{n}$ is a sequence of functions such that $\ell_{b}\left(f_{n}\right)$ are bounded for every $b$, then the sequence $\mathcal{G} f_{n}$ contains a subsequence which converges a.e. (relative to $d r d x)$.

2.2.C. Let

$$
\theta=\sup _{x \in D} c^{*}(x)
$$

where

$$
c^{*}=\sum_{i, j=1}^{d} \nabla_{i} \nabla_{j} a_{i j}-\sum_{i=1}^{d} \nabla_{i} b_{i} .
$$

Then

$$
\int_{D} f \operatorname{sign} G f d x \geq-\theta\|G f\|
$$

for all $f \in L^{1}(D)$.

Properties 2.2.A and 2.2.A* follow from well-known bounds for $g(x, y)$ ([24, Chapter 3]) and $p_{t}(x, y)([19$, Chapter 1]).

Proof of 2.2.B. Denote by $\varphi_{\delta}$ a function equal to 0 for $|t|<\delta / 2$, equal to 1 for $|t|>\delta$ and linear on $[-\delta,-\delta / 2]$ and on $[\delta / 2, \delta]$. Formula

$$
\mathcal{G}_{\delta} f(t, x ; s, y)=\varphi_{\delta}(t-s) p_{t-s}(x, y)
$$

defines a continuous kernel on $S_{b}=[0, b] \times \bar{D}$. The corresponding operator $\mathcal{G}_{\delta}$ is compact in $L^{1}\left(S_{b}\right)$ because functions $\mathcal{G}_{\delta} f_{n}$ are equicontinuous for every sequence $f_{n}$ bounded in $L^{1}\left(S_{b}\right)$.

By 2.2.A* and Fubini's theorem,

$$
\begin{aligned}
\ell_{b}\left(\mathcal{G} f-\mathcal{G}_{\delta} f\right) & =\int_{S_{b}} d t d x \int_{S_{b}}\left[1-\varphi_{\delta}(t-s)\right] p_{t-s}(x, y)|f(s, y)| d s d y \\
& \leq \int_{S_{b}} d s d y|f(s, y)| \int_{s}^{(s+\delta) \wedge b} d t d x p_{t-s}(x, y) \leq C \delta \ell_{b}(f) .
\end{aligned}
$$


Therefore $\mathcal{G}$ is a compact operator in $L^{1}\left(S_{b}\right)$. We get 2.2.B by the diagonal procedure.

Proof of 2.2.C. $1^{\circ}$. Suppose that $\varphi$ is a bounded increasing continuously differentiable function on $\mathbb{R}$ such that $\varphi(0)=0$. Suppose that

$$
u \in C^{2}(\bar{D}), \quad u=0 \quad \text { on } \partial D .
$$

Put $\Phi(t)=\int_{0}^{t} \varphi(s) d s$. By integration by parts, we get

$$
\begin{aligned}
-\int_{D} \varphi(u) L u d x & =\int_{D}\left[\sum_{i, j} a_{i j} \varphi^{\prime}(u) \nabla_{i} u \nabla_{j} u+\sum_{i}\left(\sum_{j} \nabla_{j} a_{i j}+b_{i}\right) \varphi(u) \nabla_{i} u\right] d x \\
& =\int_{D}\left[\sum a_{i j} \varphi^{\prime}(u) \nabla_{i} u \nabla_{j} u-c^{*} \Phi(u)\right] d x
\end{aligned}
$$

and therefore

$$
-\int_{D} d x \varphi(u) L u \geq-\theta \int_{D} \Phi(u) d x .
$$

$2^{\circ}$. Suppose $u=G f$ with $f \in C^{2}$. Then $u$ satisfies (2.19) and $L u=-f$. By $(2.21)$,

$$
\int_{D} \varphi(u) f d x \geq-\theta \int_{D} \Phi(u) d x
$$

An arbitrary $f \in L^{1}(D)$ is the strong limit of a sequence $f_{n} \in L^{1}(D) \cap C^{2}$. Let $u_{n}=G f_{n}, u=G f$. We have

$$
\int \varphi(u) f d x-\int \varphi\left(u_{n}\right) f_{n} d x=\int \varphi\left(u_{n}\right)\left(f-f_{n}\right) d x+\int\left(\varphi(u)-\varphi\left(u_{n}\right)\right) f d x .
$$

By 2.2.A, $u_{n} \rightarrow u$ in $L^{1}(D)$. Therefore a subsequence $u_{n_{k}}$ converges to $u$ a.e. and the second term in the right side of (2.23) converges to 0 along this subsequence. The first term also converges to 0 . Since (2.22) holds for $f_{n}$, it holds also for $f$.

$3^{\circ}$. By applying (2.22) to a sequence of functions $\varphi_{n}$ which converge boundedly to sign $u$ and by passing to the limit, we get

$$
\int_{D} f \operatorname{sign} u d x \geq-\theta \int_{D}|u| d x
$$

which is equivalent to (2.18).

2.3. Existence. Suppose that $\xi$ is an $L$-diffusion stopped at the first exit time $\tau$ from a bounded smooth domain $D, L$ satisfies conditions 1.1.A-B, $p$ is defined by condition 1.2.A and $g$ is the corresponding Green's function defined by (1.12). We consider a function in $D$ defined by the formula

$$
h=G \eta+K \sigma
$$

where $\eta$ is a finite measure on $D$ and $\sigma$ is a positive bounded Borel function on $\partial D$. Put $D(h)=\{h<\infty\}, D(h, \alpha)=\left\{h+G\left(h^{\alpha}\right)<\infty\right\}$ and $\mathcal{N}(h, \alpha)=\{\mu$ : $\left.\left\langle h+G\left(h^{\alpha}\right), \mu\right\rangle=\infty\right\}$. Note that $D(h, \alpha)$ is either empty or is the complement of a $\xi$-polar set. Let $Q(h)=\mathbb{R}_{+} \times D(h)$ and $Q(h, \alpha)=\mathbb{R}_{+} \times D(h, \alpha)$. 
Theorem 2.2. Suppose that $D(h, \alpha)$ is nonempty. Then there exists $v \geq 0$ such that

$$
v+G\left(v^{\alpha}\right)=h \quad \text { on } D(h, \alpha) .
$$

Equation (2.25) determines $v$ uniquely on $D(h, \alpha)$. We have

$$
\left\|v^{\alpha}\right\| \leq 2 C \eta(D)+C_{1}(\sigma)
$$

where $C$ is defined in 2.2.A and $C_{1}(\sigma)$ does not depend on $\eta$. Let $\tilde{X}$ be an $(L, \alpha)$ superdiffusion stopped at exit from $D$ and let

$$
\begin{aligned}
& v_{\varepsilon}(x)=-\log P_{x} \exp \left\{-\left[\int_{0}^{\infty}\left\langle\rho_{\varepsilon}, \tilde{X}_{t}\right\rangle d t+\left\langle\sigma, X_{D}\right\rangle\right]\right\}, \\
& u_{\varepsilon}(t, x)=-\log P_{x} \exp \left\{-\left[\int_{0}^{t}\left\langle\rho_{\varepsilon}, \tilde{X}_{s}\right\rangle d s+\left\langle\sigma, \tilde{X}_{t}\right\rangle\right]\right\}
\end{aligned}
$$

where $\sigma=0$ in $D, \rho_{\varepsilon}$ is given by (2.11) in $D$ and it is equal to 0 on $\partial D$.

We have

$$
\lim _{\varepsilon \rightarrow 0} v_{\varepsilon}(x)=v(x) \quad \text { on } D(h, \alpha)
$$

where $v$ is the solution of (2.25) and $u$ is the solution of the equation

$$
u+\mathcal{G}\left(u^{\alpha}\right)=\mathcal{G} \eta+\mathcal{K} \sigma \quad \text { on } Q(h, \alpha) .
$$

Moreover, if $\mu \notin \mathcal{N}(h, \alpha)$, then

$$
\left\langle v_{\varepsilon}, \mu\right\rangle \rightarrow\langle v, \mu\rangle
$$

and

$$
\left\langle u_{\varepsilon}^{t}, \mu\right\rangle \rightarrow\left\langle u^{t}, \mu\right\rangle
$$

for all $t$.

Finally,

$$
u(t, x) \uparrow v(x) \quad \text { as } \quad t \rightarrow \infty \quad \text { on } Q(h, \alpha) .
$$

Proof. By Theorems 2.1 and 2.1*, each of the equations (2.25) and (2.31) has no more than one solution. We split the proof of Theorem 2.2 into three steps. First, we establish a bound for $\left\|v_{\varepsilon}^{\alpha}\right\|$. Then we use this bound to prove formulae (2.30) and (2.33). Finally, we establish (2.34), (2.29), (2.32), (2.25), (2.31) and (2.26). 
$1^{\circ}$. It follows from (2.12) that

$$
h_{\varepsilon} \leq h \quad \text { and } h_{\varepsilon} \uparrow h \quad \text { as } \varepsilon \rightarrow 0
$$

where $h_{\varepsilon}=G \rho_{\varepsilon}+K \sigma$. By Theorem A and (1.19), $v_{\varepsilon}$ given by (2.27) satisfies equation

$$
v_{\varepsilon}+G\left(\left[v_{\varepsilon}\right]^{\alpha}\right)=h_{\varepsilon}
$$

and

$$
w(x)=-\log P_{x} \exp \left\{-\left\langle\sigma, X_{D}\right\rangle\right\}
$$

satisfies equation

$$
w+G\left(w^{\alpha}\right)=K \sigma .
$$

Note that functions $\rho_{\varepsilon}, w$ and $K \sigma$ are bounded and

$$
v_{\varepsilon}-w=G\left(F_{\varepsilon}\right)
$$

where

$$
F_{\varepsilon}=\rho_{\varepsilon}-v_{\varepsilon}^{\alpha}+w^{\alpha}
$$

By 2.2.C,

$$
\int F_{\varepsilon} \operatorname{sign}\left(v_{\varepsilon}-w\right) d x=\int F_{\varepsilon} \operatorname{sign} G F_{\varepsilon} d x \geq-\theta\left\|v_{\varepsilon}-w\right\|
$$

and, $\operatorname{since} \operatorname{sign}\left(v_{\varepsilon}^{\alpha}-w^{\alpha}\right)=\operatorname{sign}\left(v_{\varepsilon}-w\right)$, we have

$$
\left\|v_{\varepsilon}^{\alpha}-w^{\alpha}\right\|=\int\left(v_{\varepsilon}^{\alpha}-w^{\alpha}\right) \operatorname{sign}\left(v_{\varepsilon}^{\alpha}-w^{\alpha}\right) d x \leq\left\|\rho_{\varepsilon}\right\|+\theta\left\|v_{\varepsilon}-w\right\| .
$$

By 2.2.A* and (2.11),

$$
\left\|\rho_{\varepsilon}\right\| \leq C \eta(D)
$$

Note that, if $\alpha>1$, then for every $\delta>0$, there exists a constant $C_{\delta}$ such that

$$
|b-a| \leq \delta\left|b^{\alpha}-a^{\alpha}\right|+C_{\delta}
$$

for all reals $a, b$. It follows from $(2.41),(2.42)$ and (2.43) that

$$
\left\|v_{\varepsilon}^{\alpha}-w^{\alpha}\right\| \leq \theta \delta\left\|v_{\varepsilon}^{\alpha}-w^{\alpha}\right\|+C \eta(D)+\theta C_{\delta} .
$$

If $\delta \theta \leq 1 / 2$, then

$$
\left\|v_{\varepsilon}^{\alpha}-w^{\alpha}\right\| \leq 2 C \eta(D)+2 \theta C_{\delta} .
$$


Since $w \leq K \sigma$ and $\sigma$ is bounded, (2.45) implies

$$
\left\|v_{\varepsilon}^{\alpha}\right\| \leq 2 C \eta(D)+C_{1}(\sigma)
$$

where $C_{1}(\sigma)=2 \theta C_{\delta}+\left\|(K \sigma)^{\alpha}\right\|$.

$2^{\circ}$. By (1.23), (2.12) and (2.24),

$$
\mathcal{G} \rho_{\varepsilon}+\mathcal{K} \sigma \leq G \rho_{\varepsilon}+K \sigma \leq h .
$$

By Theorem B,

$$
u_{\varepsilon}+\mathcal{G}\left(u_{\varepsilon}^{\alpha}\right)=\mathcal{G} \rho_{\varepsilon}+\mathcal{K} \sigma
$$

and

$$
W(t, x)=-\log P_{x} \exp \left\{-\left\langle\sigma, \tilde{X}_{t}\right\rangle\right\}
$$

is a solution of the equation

$$
W+\mathcal{G}\left[W^{\alpha}\right]=\mathcal{K} \sigma .
$$

We have

$$
u_{\varepsilon}-W=\mathcal{G}\left(F_{\varepsilon}\right)
$$

where

$$
F_{\varepsilon}=\rho_{\varepsilon}-u_{\varepsilon}^{\alpha}+W^{\alpha} .
$$

By (2.28) and (2.27),

$$
u_{\varepsilon}(t, x) \leq v_{\varepsilon}(x) \quad \text { for all } t, x .
$$

For every $b$, by (2.46) and (2.51),

$$
b^{-1} \ell_{b}\left[\left(u_{\varepsilon}\right)^{\alpha}\right] \leq\left\|v_{\varepsilon}^{\alpha}\right\| \leq 2 C \eta(D)+C_{1}(\sigma) .
$$

It follows from (2.11) and 1.2.B that

$$
\mathcal{G} \rho_{\varepsilon}(t, x)=\int_{\varepsilon}^{t+\varepsilon} d s \int_{D} p_{s}(x, y) \eta(d y) \leq h(x)
$$

and therefore

$$
\mathcal{G} \rho_{\varepsilon} \rightarrow \mathcal{G} \eta \quad \text { as } \quad \varepsilon \rightarrow 0 \quad \text { on } D(h) .
$$

For every $b, \ell_{b}\left(F_{\varepsilon}\right)$ are bounded by $(2.50),(2.42)$ and (2.52). By (2.49) and 2.2.B, every sequence $u_{\varepsilon_{n}}$ contains a subsequence which converges, a.e., to a limit $u$. Suppose $u_{\varepsilon_{n}} \rightarrow u$ a.e. By (2.48) and (2.47), $u_{\varepsilon} \leq h$. It follows from (1.23) and the dominated convergence theorem that

$$
\mathcal{G}\left[\left(u_{\varepsilon_{n}}\right)^{\alpha}\right] \rightarrow \mathcal{G}\left[(u)^{\alpha}\right] \quad \text { on } Q(h, \alpha) .
$$


By (2.55) and (2.48), $u$ satisfies (2.31) a.s. Formula (2.30) holds because, otherwise, $\left|u_{\varepsilon_{n}}-u\right|>\delta$ for some $(r, x) \in Q(h, \alpha), \delta>0$ and for some sequence $\varepsilon_{n} \rightarrow 0$. By applying once more the dominated convergence theorem, we get (2.33).

$3^{\circ}$. It is clear from $(2.28)$ that $u_{\varepsilon}(t, x)$ is monotone increasing in $t$. Therefore for every $x \in D(h, \alpha), u(t, x)$ is also monotone increasing in $t$. By the monotone convergence theorem, $v(x)=\lim _{t \rightarrow \infty} u(t, x)$ satisfies (2.25). Formula (2.26) follows from (2.52) and (2.34).

Note that $u_{\varepsilon} \leq v_{\varepsilon}$ and, by (2.30) and (2.34), $\liminf _{\varepsilon \rightarrow 0} v_{\varepsilon} \geq v$ on $D(h, \alpha)$. On the other hand, it follows from (1.25) and (1.13) that $\stackrel{\mathcal{G}}{\mathcal{G}}\left(u_{\varepsilon}^{\alpha}\right) \leq \mathcal{G}\left(v_{\varepsilon}^{\alpha}\right) \leq G\left(v_{\varepsilon}^{\alpha}\right)$ and, by (2.48), (2.36), (2.12), (2.53), (1.25) and (1.26),

$$
0 \leq v_{\varepsilon}-u_{\varepsilon}^{t} \leq \int_{t+\varepsilon}^{\infty} d s \int_{D} p_{s}(x, y) \eta(d y)+\Pi_{x} \sigma\left(\xi_{\tau}\right) 1_{\tau>t}
$$

and therefore $\lim \sup v_{\varepsilon} \leq v$ on $D(h, \alpha)$. Clearly, this implies (2.29). Formula (2.32) can be deduced from (2.33) in an analogous way.

\section{Proof of Theorem 1.3}

3.1. We use several times a property of exit measures which will be established in Lemma 3.1. We start from a functional

$$
B_{t}(\varepsilon)=\int_{0}^{t}\left\langle\rho_{\varepsilon}, \tilde{X}_{s}\right\rangle d s+C_{t}
$$

where $\rho_{\varepsilon}$ is given by (2.11) and $C_{t}$ is a left continuous modification of $\left\langle\sigma, \tilde{X}_{t}\right\rangle$ which we define in Lemma 3.2. Put

$$
B_{t}=\operatorname{limmed}_{k \rightarrow \infty} B_{t}(1 / k) \quad \text { for all } t>0
$$

where lim med is Mokobodzki's medial limit. It is defined for every sequence $a_{n} \in$ $[0, \infty]$ and it takes values in $[0, \infty]$. We need the following properties of this limit (see, e.g., $[4$, X.56, X.57]):

3.1.A. $\liminf a_{n} \leq \lim \operatorname{med} a_{n} \leq \lim \sup a_{n}$;

3.1.B. $\lim \operatorname{med}\left(a_{n}+b_{n}\right)=\lim \operatorname{med} a_{n}+\lim \operatorname{med} b_{n}$;

3.1.C. If $a_{n} \leq b_{n}$ for all $n$, then $\lim \operatorname{med} a_{n} \leq \lim \operatorname{med} b_{n}$;

3.1.D. Let $Z_{n}$ be measurable mappings from a measurable space $(\Omega, \mathcal{F})$ to $[0, \infty]$. Then $Z(\omega)=\lim$ med $Z_{n}(\omega)$ is measurable with respect to the universal completion of $\mathcal{F}$. If $P$ is a probability measure on $(\Omega, \mathcal{F})$ and if $Z_{n} \rightarrow Y$ in $P$-probability, then $Y=Z P$-a.s.

In Theorem 3.1, we construct a functional $B$ of an $(L, \alpha)$-superdiffusion $\tilde{X}$ stopped at the exit from a bounded smooth domain $D$ which satisfies conditions 1.6.A, B and the following condition:

$$
B_{s+t} \leq B_{s}+\theta_{s} B_{t} \quad \text { a.s. for every } s, t .
$$

Moreover, for every $\mu \notin \mathcal{N}(h, \alpha)$ :

$$
B_{t}=\lim _{\varepsilon \rightarrow 0} B_{t}(\varepsilon) \quad \text { in } P_{\mu} \text {-probability for all } t \in \mathbb{R}_{+}
$$


and

$$
-\log P_{\mu} e^{-B_{t}}=\left\langle u^{t}, \mu\right\rangle
$$

where $u$ satisfies (2.31).

The next step is a passage to the limit from bounded smooth domains to an arbitrary domain $E$. We assume that $h$ is given by (1.17) and that $E(h, \alpha)=\{h<$ $\left.\infty, G\left(h^{\alpha}\right)<\infty\right\}$ is nonempty. We consider a sequence of bounded smooth domains $D_{n}$ which approximate $E$ and we denote by $G^{n}, K^{n}$ the Green and Poisson operators corresponding to the $L$-diffusion stopped at the exit from $D_{n}$. Put $\sigma_{n}=1_{E \backslash D_{n}} K \nu$ and denote by $B^{n}$ the function corresponding to

$$
h_{n}(x)=\int_{D_{n}} g^{n}(x, y) \eta(d y)+K^{n} \sigma_{n}(x)
$$

by Theorem 3.1. By 3.1.C, D, function

$$
B_{t}=\operatorname{limmed}_{n \rightarrow \infty} B_{t}^{n}
$$

satisfies 1.6.A, B. We show that, for every $\mu \notin \mathcal{N}(h, \alpha)$ and every $t$,

$$
B_{t}=\lim _{n \rightarrow \infty} B_{t}^{n} \quad P_{\mu}-\text { a.s. }
$$

Function $A_{t}=B_{t-}$ satisfies 1.6.A-C and 1.6.D** with $\mathcal{N}=\mathcal{N}(h, \alpha)$.

At the final stage, we use Lemma 3.3 to decompose measures $\eta, \nu$, subject to condition (1.22), into series of measures $\eta_{n}, \nu_{n}$ for which $E\left(h_{n}, \alpha\right) \neq \emptyset$ (here $\left.h_{n}=G \eta_{n}+K \nu_{n}\right)$. The functional corresponding to $\eta, \nu$ is defined as the sum of functionals corresponding to $\eta_{n}, \nu_{n}$.

This way we obtain a functional of $X$, subject to conditions 1.6.A, B, C, for which 1.6. $\mathrm{D}^{* *}$ and (1.45) hold for all $\mu$ outside of an exceptional set $\mathcal{N}$. Then we refer to a result in [4] to prove the existence of an equivalent functional which satisfies 1.6.D*.

\subsection{A property of exit measures.}

Lemma 3.1. Suppose that $Q_{1} \supset Q_{2}$ are open subsets of $S$ and $\Gamma \cap Q_{1}=\emptyset$. Then $X_{Q_{1}}(\Gamma) \geq X_{Q_{2}}(\Gamma)$ a.s.

Proof. For every $\nu \in \mathcal{M}(E), P_{\nu}\left\{X_{Q_{1}}(\Gamma) \geq \nu(\Gamma)\right\}=1$. Indeed, $\Pi_{r, x}\left\{\tau^{r}=r\right\}=1$ for every $(r, x) \notin Q_{1}$ and, by (1.32), for every $\lambda>0$,

$$
P_{\nu} e^{-\lambda X_{Q_{1}}(\Gamma)}=e^{-\left\langle v_{\lambda}, \nu\right\rangle}
$$

with $v_{\lambda}=\lambda 1_{\Gamma}$ on $\Gamma$. Hence,

$$
P_{\nu} e^{-\lambda X_{Q_{1}}(\Gamma)} \leq e^{-\lambda \nu(\Gamma)} .
$$

Put $Y=X_{Q_{1}}(\Gamma)-\nu(\Gamma)$. By (3.9), $P_{\nu} e^{-\lambda Y} \leq 1$ for all $\lambda>0$ and therefore $Y \geq 0$ $P_{\nu}$-a.e.

It follows from (1.34) that, for every positive measurable $f, P_{\mu} f\left(X_{Q_{2}}, X_{Q_{1}}\right)=$ $P_{\mu} F\left(X_{Q_{2}}\right)$ where $F(\nu)=P_{\nu} f\left(\nu, X_{Q_{1}}\right)$. If $f\left(\nu_{1}, \nu_{2}\right)=1_{\nu_{1}(\Gamma) \leq \nu_{2}(\Gamma)}$, then $F(\nu)=$ $P_{\nu}\left\{\nu(\Gamma) \leq X_{Q_{1}}(\Gamma)\right\}=1$. 
3.3. Regularization of $\left\langle\sigma, \tilde{X}_{t}\right\rangle$.

Lemma 3.2. Let $\tilde{X}$ be an $(L, \alpha)$-superdiffusion stopped at the exit from a bounded smooth domain $D$ and let $\sigma$ be a positive Borel function on $\bar{D}$ which vanishes on $D$. There exists a function $C_{t}$ subject to conditions 1.6.A-C such that, for every $t$,

$$
C_{t}=\left\langle\sigma, \tilde{X}_{t}\right\rangle \quad \text { a.s. }
$$

Proof. Put $Y_{t}=\left\langle\sigma, \tilde{X}_{t}\right\rangle$. Recall (see Section 1.5) that $\tilde{X}_{t}=X_{Q_{t}}$ where $Q_{t}=$ $[0, t) \times D$. It follows from Lemma 3.1 that $\tilde{X}_{r}(\Gamma) \leq \tilde{X}_{s}(\Gamma)$ a.s. if $r<s$ and $\Gamma \cap Q_{s}=\emptyset$. Since $\sigma=0$ in $Q_{s}, Y_{r} \leq Y_{s}$ a.s. Denote by $\mathbb{Q}_{+}$the set of positive rationals. The set

$$
\Omega_{t}=\left\{Y_{r} \leq Y_{s} \text { for all } r<s \in \mathbb{Q}_{+} \cap[0, t)\right\}
$$

belongs to $\mathcal{F}_{t}$ and $P_{\mu}\left\{\Omega_{t}\right\}=1$ for all $\mu \in \mathcal{M}(D)$. Function

$$
C_{t}= \begin{cases}\lim _{s \uparrow t, s \in \mathbb{Q}_{+}} Y_{s} & \text { on } \Omega_{t}, \\ \infty & \text { otherwise }\end{cases}
$$

satisfies conditions 1.6.A-C. It remains to prove that $Y_{t}=C_{t}$ a.s. By Theorem B,

$$
-\log P_{\mu} e^{-Y_{t}}=\left\langle u^{t}, \mu\right\rangle
$$

where $u$ is a solution of the equation

$$
u+\mathcal{G}\left[u^{\alpha}\right]=\mathcal{K} \sigma .
$$

By $(3.11), u(t, x)$ is monotone increasing in $t$. Put $u_{-}(t, x)=u(t-, x)$. Since $\Pi_{x}\{\tau=t\}=0$ for all $t$, function $\mathcal{K} \sigma$ is continuous in $t$. By passing to the limit in (3.12), we get

$$
u_{-}+\mathcal{G}\left[u_{-}^{\alpha}\right]=\mathcal{K} \sigma .
$$

By (1.25), functions $\mathcal{K} \sigma \leq K \sigma$ are bounded and, by Theorem $2.1^{*}, u_{-}=u$. By (3.11), $P_{\mu} e^{-Y_{t}}=P_{\mu} e^{-C_{t}}$. Since $Y_{t} \leq C_{t}$, this implies (3.10).

3.4 .

Lemma 3.3. Let $\eta$ and $\nu$ satisfy condition (1.22). Then there exist measures $\eta_{n}, \nu_{n}$ such that

$$
\eta=\eta_{1}+\cdots+\eta_{n}+\cdots, \quad \nu=\nu_{1}+\cdots+\nu_{n}+\cdots
$$

and

$$
G\left(h_{n}^{\alpha}\right)(c)<\infty
$$

where $h_{n}=G \eta_{n}+\mathcal{K} \nu_{n}$ and $c$ is the same as in formula (1.16).

Proof. Since $\left(\frac{a+b}{2}\right)^{\alpha} \leq \frac{1}{2}\left(a^{\alpha}+b^{\alpha}\right)$ for all $\alpha, a, b \geq 0$, we can assume that $\eta=0$ or $\nu=0$. We refer to [18, Theorem 2.2] in the first case and [2, Lemma 4.2] in the second case. 
3.5 .

Theorem 3.1. Let $\tilde{X}$ be an $(L, \alpha)$-superdiffusion stopped at the exit from a bounded smooth domain $D$ and let $h, \eta, \rho_{\varepsilon}$ and $\sigma$ be as in Theorem 2.2. If $B_{t}(\varepsilon)$ is defined by (3.1), then function $B_{t}$ given by (3.2) satisfies conditions 1.6.A, B, (3.3), (3.4) and (3.5).

Proof. Properties 1.6.A, B follow from 3.1.C, D. By Theorem B,

$$
u_{\delta \varepsilon}(t, x)=-\log P_{x} \exp \left\{-\frac{1}{2}\left(B_{t}(\delta)+B_{t}(\varepsilon)\right)\right\}
$$

satisfies the equation

$$
u_{\delta \varepsilon}+\mathcal{G}\left[u_{\delta \varepsilon}^{\alpha}\right]=\frac{1}{2}\left(\mathcal{G} \rho_{\delta}+\mathcal{G} \rho_{\varepsilon}\right)+\mathcal{K} \sigma
$$

The same arguments as in the proof of Theorem 2.2 show that, for all $\mu \notin \mathcal{N}(h, \alpha)$ and all $t$,

$$
\left\langle u_{\delta \varepsilon}^{t}, \mu\right\rangle \rightarrow\left\langle u^{t}, \mu\right\rangle \quad \text { as } \delta, \varepsilon \rightarrow 0
$$

where $u$ is the unique solution of (2.31).

By Theorem B,

$$
P_{\mu}\left[e^{-B_{t}(\varepsilon) / 2}-e^{-B_{t}(\delta) / 2}\right]^{2}=e^{-\left\langle u_{\varepsilon \varepsilon}^{t}, \mu\right\rangle}+e^{-\left\langle u_{\delta \delta}^{t}, \mu\right\rangle}-2 e^{-\left\langle u_{\delta \varepsilon}^{t}, \mu\right\rangle}
$$

for every $\mu \in \mathcal{M}(D)$. If $\mu \notin \mathcal{N}(h, \alpha)$, then, by (3.15), the right side in (3.16) tends to 0 as $\delta, \varepsilon \rightarrow 0$. Hence $e^{-B_{t}(\varepsilon)}$ converges in $L^{2}\left(P_{\mu}\right)$ as $\varepsilon \rightarrow 0$ which implies that $B_{t}(\varepsilon)$ converges in $P_{\mu}$-probability to a limit $B_{t}^{\mu}$. It follows from 3.1.D that $B_{t}=B_{t}^{\mu}$ $P_{\mu}$-a.s. which implies (3.4). To prove (3.3), we note that $\tilde{X}_{t}=X_{Q(t)}$ where $Q(t)=$ $[0, t) \times D$. Therefore (see Section 1.5)) $\theta_{s} \tilde{X}_{t}=X_{Q(s, t)}$ where $Q(s, t)=S_{<s} \cup Q(s+t)$ and, by Lemma $3.1,\left\langle\rho_{\varepsilon}, \theta_{s} \tilde{X}_{t}\right\rangle \geq\left\langle\rho_{\varepsilon}, \tilde{X}_{s+t}\right\rangle$ and $\left\langle\sigma, \theta_{s} \tilde{X}_{t}\right\rangle \geq\left\langle\sigma, \tilde{X}_{s+t}\right\rangle$ a.s. Clearly, restrictions of measures $\tilde{X}_{s}$ and $\tilde{X}_{s+t}$ to $[0, s) \times \partial D$ coincide, and, by (3.1),

$$
B_{s}(\varepsilon)+\theta_{s} B_{t}(\varepsilon) \geq B_{s+t}(\varepsilon) \quad \text { a.s. }
$$

and (3.3) follows from (3.2).

Let $\mu \notin \mathcal{N}(h, \alpha)$. By (1.42),

$$
-\log P_{\mu} e^{-B_{t}(\varepsilon)}=\left\langle u_{\varepsilon}^{t}, \mu\right\rangle
$$

where $u_{\varepsilon}$ is given by (2.28). By (3.4) and (2.33), this implies (3.5).

3.6. The next step in the program outlined in Section 3.1 is a passage to the limit from bounded smooth domains to an arbitrary domain $E$. Recall that, according to Section 1.2, $L$-diffusion $\xi$ in $E$ can be constructed by using a sequence of bounded smooth domains $D_{n}$ such that $\bar{D}_{n} \subset D_{n+1}$ and $E=\bigcup D_{n}$ : the transition density $p_{t}(x, y)$ of $\xi$ is the limit of monotone increasing sequence $p_{t}^{n}(x, y)$ defined in 1.2.A (it is convenient to set $p_{t}^{n}(x, y)=0$ if $x \notin D_{n}$ or $\left.y \notin D_{n}\right)$. Green's functions $g^{n}(x, y)$, 
$g(x, y)$ and Green's operators $G^{n}, G$ corresponding to $p^{n}, p$ are determined by (1.12) and (1.13). Operators $K^{n}$ correspond by (1.26) to the first exit times $\tau_{n}$ from $D_{n}$.

Let $X$ be an $(L, \alpha)$-superdiffusion in $E$ and let $X^{n}$ be an $(L, \alpha)$-superdiffusion stopped at the exit from $D_{n}$. Denote by $Y_{t}^{n}$ the restriction of $X_{t}^{n}$ to $D_{n}$. By Lemma 3.1 , for every $t$ and every $n$,

$$
Y_{t}^{n} \leq Y_{t}^{n+1} \quad \text { a.s. }
$$

By (1.37),

$$
P_{\mu} Y_{t}^{n}(B)=\int_{E} \mu(d x) \int_{B} p_{t}^{n}(x, y) d y \uparrow \int_{E} \mu(d x) \int_{B} p_{t}(x, y) d y=P_{\mu} X_{t}(B)
$$

and therefore

$$
Y_{t}^{n} \uparrow X_{t} \quad \text { a.s. }
$$

3.7. Let $h$ be given by (1.17) with finite measures $\eta$ and $\nu$. Suppose that $E(h, \alpha) \neq$ $\emptyset$. Put $f=K \nu$. By (1.19) and (1.26),

$$
f(x)=\Pi_{x} \sigma_{n}\left(\xi_{\tau_{n}}\right)=K^{n} \sigma_{n}(x)
$$

where $\sigma_{n}=1_{E \backslash D_{n}} f$. We define $B^{n}$ and $B$ as in Section 3.1. By 3.1.D, to prove formula (3.8), we need only show that $B_{t}^{n}$ converges $P_{\mu}$-a.s. as $n \rightarrow \infty$. Put

$$
Z_{t}^{n}(\varepsilon)=\int_{0}^{t}\left\langle\rho_{\varepsilon}^{n}, Y_{r}^{n}\right\rangle d r
$$

By 3.1.B,

$$
B_{t}^{n}=Z_{t}^{n}+C_{t}^{n}
$$

where

$$
Z_{t}^{n}=\operatorname{limmed}_{k \rightarrow \infty} Z_{t}^{n}(1 / k) .
$$

For every $n, \rho_{\varepsilon}^{n+1} \geq \rho_{\varepsilon}^{n}$ and, by (3.20) and (3.18), $Z_{t}^{n}(\varepsilon)$ is, a.s., monotone increasing in $n$. By 3.1.C, sequence $Z_{t}^{n}$ has the same property and therefore it converges $P_{\mu^{-}}$ a.s.

On the other hand, since $f$ is $L$-harmonic, it follows from the Markov property (1.34) that the sequence $W_{n}=\left\langle f, X_{t}^{n}\right\rangle$ is a martingale with respect to $P_{\mu}$. Therefore $C_{t}^{n}$ also converges a.s.

3.8. Put $S(h, \alpha)=\mathbb{R}_{+} \times E(h, \alpha)$. By Theorem 2.2 , for every $\mu \notin \mathcal{N}(h, \alpha)$,

$$
-\log P_{\mu} e^{-B_{t}^{n}}=\int_{D_{n}} u_{n}(t, x) \mu(d x)
$$

where $u_{n}$ satisfies the equation

$$
u_{n}(t, x)+\int_{0}^{t} d s \int_{D_{n}} p_{t-s}^{n}(x, y) u_{n}(s, y)^{\alpha} d y=H_{n}(t, x) \quad \text { on } S(h, \alpha)
$$


with

$$
H_{n}(t, x)=\int_{0}^{t} d s \int_{D_{n}} p_{t-s}^{n}(x, y) \eta(d y)+\Pi_{x} f\left(\xi_{\tau_{n}}\right) 1_{\tau_{n}<t} .
$$

Moreover, by (2.28), (2.30) and (3.20),

$$
u_{n}(t, x)=-\lim _{\varepsilon \rightarrow 0} \log P_{x} \exp \left\{-B_{t}^{n}(\varepsilon)\right\} \quad \text { on } S(h, \alpha) .
$$

By (3.4),

$$
u_{n}(t, x)=-\log P_{x} e^{-B_{t}^{n}} \quad \text { on } S(h, \alpha) .
$$

By (3.8),

$$
u_{n}(t, x) \rightarrow u(t, x)=-\log P_{x} e^{-B_{t}} \quad \text { on } S(h, \alpha) .
$$

Note that

$$
\Pi_{x} f\left(\xi_{\tau_{n}}\right) 1_{\tau_{n}<t}=f(x)-\Pi_{x} f\left(\xi_{t}\right) 1_{\tau_{n} \geq t}
$$

and therefore $H_{n}$ converges to

$$
H(t, x)=\int_{0}^{t} d s \int_{E} p_{t-s}(x, y) \eta(d y)+F(t, x)
$$

where

$$
F(t, x)=f(x)-\Pi_{x} f\left(\xi_{t}\right) .
$$

By (3.21), (3.22) and (1.17), $u_{n} \leq h$. The second term in (3.21) converges to $\mathcal{G}\left[u^{\alpha}\right]$ by (2.2) and the dominated convergence theorem. Hence, (3.21) implies

$$
u+\mathcal{G}\left[u^{\alpha}\right]=H \quad \text { on } S(h, \alpha) .
$$

3.9. Note that $u(t, x)$ increases in $t$ by (3.24) and 1.6.A. Put $u_{-}(t, x)=u(t-, x)$. An $L$-excessive function $f$ has a representation

$$
f=f_{0}+\int_{0}^{\infty} \varphi_{s} d s
$$

where $T_{t} f_{0}=f_{0}$ and $T_{t} \varphi_{s}=\varphi_{s+t}$ for all $t, s$ (see [10, Section 2.8]). Therefore

$$
H(t, x)=\int_{0}^{t} d s\left[\int_{E} p_{s}(x, y) \eta(d y)+\varphi_{s}\right]
$$

is increasing and continuous in $t$. By passing to the limit in (3.24) and (3.27), we get

$$
\begin{gathered}
u_{-}(t, x)=-\log P_{x} e^{-B_{t-}}, \\
u_{-}+\mathcal{G}\left[u_{-}^{\alpha}\right]=H \quad \text { on } S(h, \alpha) .
\end{gathered}
$$


By Theorem 2.1*, this implies $u_{-}=u$. Since $B_{t-} \leq B_{t},(3.24)$ and (3.28) yield $B_{t-}=B_{t}$ a.s. Function $A_{t}=B_{t-}$ satisfies conditions 1.6.A-C.

We claim that 1.6. $\mathrm{D}^{* *}$ holds for $\mathcal{N}=\mathcal{N}(h, \alpha)$. Indeed, if $\mu \notin \mathcal{N}(h, \alpha)$, then

$$
B_{t}^{n}(\varepsilon)=Z_{t}^{n}(\varepsilon)+C_{t}^{n}
$$

converges in $P_{\mu}$-probability to $B_{t}^{n}$ by (3.4). By the Markov property (1.34),

$$
P_{\mu} e^{-B_{s+t}^{n}(\varepsilon)}=P_{\mu}\left[e^{-B_{s}^{n}(\varepsilon)} P_{Y_{s}^{n}} e^{-B_{t}^{n}(\varepsilon)}\right]
$$

for all $s, t>0$. This implies

$$
P_{\mu} e^{-B_{s+t}^{n}}=P_{\mu}\left[e^{-B_{s}^{n}} P_{Y_{s}^{n}} e^{-B_{t}^{n}}\right]
$$

and therefore

$$
\left|P_{\mu} e^{-B_{s+t}^{n}}-P_{\mu}\left[e^{-B_{s}^{n}} P_{X_{s}} e^{-B_{t}^{n}}\right]\right| \leq P_{\mu}\left|P_{Y_{s}^{n}} e^{-B_{t}^{n}}-P_{X_{s}} e^{-B_{t}^{n}}\right|
$$

By (3.5), the right side is equal to

$$
P_{\mu}\left|e^{-\left\langle v_{n}^{t}, Y_{s}^{n}\right\rangle}-e^{-\left\langle v_{n}^{t}, X_{s}\right\rangle}\right|
$$

where $v_{n}^{t}(x)=u_{n}^{t}(0, x)$. By (3.7), $v_{n}^{t} \leq h$ and therefore (3.29) does not exceed

$$
P_{\mu}\left|1-e^{-\left\langle h, X_{s}-Y_{s}^{n}\right\rangle}\right| .
$$

By (3.19), this tends to 0 and we conclude from (3.29) and the Markov property of $X$ that

$$
P_{\mu} e^{-B_{s+t}}=P_{\mu} e^{-B_{s}} P_{X_{s}} e^{-B_{t}}=P_{\mu} e^{-\left(B_{s}+\theta_{s} B_{t}\right)} .
$$

By (3.1), $B_{s+t} \leq B_{s}+\theta_{s} B_{t}$ and (3.30) implies 1.6.D**.

We get (1.45) by passing to the limit in (3.5) and (3.27) as $t \rightarrow \infty$.

3.10. Let $h$ be an arbitrary function of the form (1.17) with $\eta$ and $\nu$ subject to conditions (1.22). Consider measures $\eta_{n}$ and $\nu_{n}$ defined in Lemma 3.3. Denote by $A^{n}$ the functional of $X$ corresponding to $h_{n}(x)=G \eta_{n}+K \nu_{n}$ by Section 3.9 and put

$$
A=A_{1}+\cdots+A_{n}+\cdots .
$$

Clearly, conditions 1.6.A,B,C and 1.6.D** hold for $A$. Formula (1.45) holds if $\mu \notin \mathcal{N}=\bigcup \mathcal{N}\left(h_{n}, \alpha\right)$ and $v$ satisfies (1.14) on $E=\bigcap E\left(h_{n}, \alpha\right)$. Function $\tilde{v}$ defined by (1.15) is a solution of (1.14) everywhere. It also satisfies (1.45).

Formula (1.44) can be obtained from (1.45) in the same way as (1.37) was deduced from (1.35).

By the "perfection" theorem $[4,15.8]$, there exists a functional equivalent to $A$ which satisfies 1.6.D*. (In [4] functionals without an exceptional set are considered, but the proof is applicable without any change to our case.) 


\section{Proof of Theorems 1.2 And 1.4}

4.1.

Lemma 4.1. Let an excessive function be given by formula (1.17). If there exists $u$ such that

$$
u+G\left(u^{\alpha}\right)=h
$$

then there exists $v$ such that

$$
v+G\left(v^{\alpha}\right)=K \nu
$$

Proof. Let $D_{n}, G^{n}$ and $K^{n}$ have the same meaning as in Section 3.6. By the strong Markov property of $\xi$, (4.1) implies

$$
u+G^{n}\left(u^{\alpha}\right)=G^{n} \eta+K^{n} u \quad \text { on } D_{n} .
$$

By Theorem A,

$$
v_{n}(x)=-\log P_{x} e^{-\left\langle u, X_{D_{n}}\right\rangle}
$$

satisfies the equation

$$
v_{n}+G^{n}\left(v_{n}^{\alpha}\right)=K^{n} u \quad \text { on } D_{n}
$$

We use again the strong Markov property of $\xi$ to get from here that, for each $m>n$,

$$
v_{m}+G^{n}\left(v_{m}^{\alpha}\right)=K^{n} v_{m} \quad \text { on } D_{n} .
$$

We conclude from Theorem 2.1, by comparing (4.3) and (4.4), that $v_{n} \leq u$ in $D_{n} \cap E(h)$, and, by comparing (4.4) and (4.5), that $v_{m} \leq v_{n}$ in $D_{n} \cap E(h)$. Therefore there exists a limit

$$
v=\lim _{n \rightarrow \infty} v_{n} \quad \text { on } E(h) .
$$

It follows from (4.3) by monotone convergence theorem that

$$
u+G\left(u^{\alpha}\right)=G \eta+\lim K^{n} u .
$$

In combination with (4.1), this yields $\lim K^{n} u=K \nu$ on $E(h)$. By (4.1), $G\left(u^{\alpha}\right)<$ $\infty$ on $E(h)$ and, by the dominated convergence theorem, $\lim G^{n}\left(v_{n}^{\alpha}\right)=G\left(v^{\alpha}\right)$. Therefore (4.4) implies that (4.2) holds on $E(h)$. It holds everywhere for a function $v$ modified by formula (1.15).

4.2. Proof of Theorem 1.2. Suppose that $u$ is a solution of (4.1). By Lemma 4.1 , equation (4.2) has a solution and $\nu$ does not charge $\mathcal{R}^{*}$-polar sets by Theorem 3.1 in [18].

It remains to prove that $\eta(\Gamma)=0$ for $\mathcal{R}$-polar sets $\Gamma$. We can assume that $\Gamma$ is compact. Let $D$ be a bounded smooth domain such that $\Gamma \subset D$ and $\bar{D} \subset E$. Equation (4.1) implies

$$
u+G_{D}\left(u^{\alpha}\right)=G_{D} \eta+K_{D} u \quad \text { in } \quad D
$$


(cf. (4.3)). By Theorem $\mathrm{E}^{\circ}$ in [17], $\operatorname{Cap}_{2, \alpha^{\prime}}(\Gamma)=0$. We use the following fact (see Lemma 4.1 in [2]): a signed measure $\gamma$ does not charge sets $\Gamma$ with $\operatorname{Cap}_{2, \alpha^{\prime}}(\Gamma)=0$ if

$$
\int_{D} \varphi(x) \gamma(d x) \leq \text { const. }\|\varphi\|_{2, \alpha^{\prime}} \quad \text { for all } \varphi \in C_{0}^{\infty}(D)
$$

(here $\|\varphi\|_{2, \alpha^{\prime}}$ is the norm of $\varphi$ in the Sobolev space $W^{2, \alpha^{\prime}}(D)$ ).

By Lemma 4.1, there exists $v \geq 0$ such that

$$
v+G_{D}\left(v^{\alpha}\right)=K_{D} u \quad \text { in } D .
$$

By Theorem 2.1, $w=u-v \geq 0$. There exists a function $q \geq 0$ such that $u^{\alpha}-v^{\alpha}=$ $q w$ a.e. (cf. proof of Theorem $2.1^{*}$ ). It follows from (4.6) and (4.7) that

$$
w+G_{D}(q w)=G_{D} \eta .
$$

Let $\gamma(d x)=\eta(d x)-(q w)(x) d x$ and $\varphi \in C_{0}^{\infty}(D)$. Put $\psi=-L^{*} \varphi$. Note that $\|\psi\|_{\alpha^{\prime}} \leq\|\varphi\|_{2, \alpha^{\prime}}$ and

$$
\varphi(y)=\int_{D} d x \psi(x) g_{D}(x, y)
$$

By (4.8), $G_{D} \gamma=w$ and therefore

$$
\begin{aligned}
\int_{D} \varphi(x) \gamma(d x) & =\int_{D \times D} d x \psi(x) g_{D}(x, y) \gamma(d y)=\int_{D} w(x) \psi(x) d x \\
& \leq\|w\|_{\alpha}\|\psi\|_{\alpha^{\prime}} \leq\|w\|_{\alpha}\|\varphi\|_{2, \alpha^{\prime}} .
\end{aligned}
$$

If $h(c)<\infty$, then $G\left(u^{\alpha}\right)(c)<\infty$ by $(4.1)$ and $u \in L^{\alpha}(D)$ because $\inf _{D} g(c, y)>0$. We have $0 \leq w \leq u$ and therefore $w \in L^{\alpha}(D)$. Hence $\gamma(\Gamma)=0$. Since $\operatorname{Cap}_{2, \alpha^{\prime}}(\Gamma)=$ 0 implies that the Lebesgue measure of $\Gamma$ is equal to 0 , we get $\eta(\Gamma)=0$.

4.3. Localization. To prove Theorem 1.4, we need some preparations. Suppose that $h$ is the potential of a linear additive functional $A$ with exceptional set $\mathcal{N}$ and let $\eta, \nu$ correspond to $h$ by (1.17). For every positive bounded continuous function $\varphi$ on $E \cup E^{*}$, we put $h^{\varphi}(x)=G\left(\eta^{\varphi}\right)+K\left(\nu^{\varphi}\right)$ where $\eta^{\varphi}(d x)=\varphi(x) \eta(d x), \nu^{\varphi}(d x)=$ $\varphi(x) \nu(d x)$. It follows from 1.6. ${ }^{*}$, the strong Markov property of $X$ and (1.44) that

$$
\left\langle h, X_{T}\right\rangle=P_{\mu}\left\{A_{\infty} \mid \mathcal{F}_{T}\right\}-A_{T} \quad P_{\mu} \text {-a.s. }
$$

for every $\mathcal{F}_{t}$-stopping time $T$ and for every $\mu \notin \mathcal{N}$. It is easy to see from here that $\left\langle h, X_{t}\right\rangle$ is a supermartingale of class (D) relative to $P_{\mu}$ (cf. [4, V.15]). Since $\left\langle h^{\varphi}, X_{t}\right\rangle \leq$ const $\left\langle h, X_{t}\right\rangle$, the same is true for $\left\langle h^{\varphi}, X_{t}\right\rangle$. By [4, Th. XV.6] or [25, Th. $38.1]^{13}$, there exists a natural additive functional $A^{\varphi 14}$ such that:

4.3.A. $P_{\mu} A_{\infty}^{\varphi}=\left\langle h^{\varphi}, \mu\right\rangle$ for all $\mu \notin \mathcal{N}$.

We call it the $\varphi$-localization of $A$. In the same way as in Theorem 3.3 of [17], we establish:

4.3.B. If $\varphi_{1} \leq \varphi_{2}$, then $A^{\varphi_{1}} \leq A^{\varphi_{2}} P_{\mu}$-a.s. for all $\mu \notin \mathcal{N}$.

4.3.C. If $\varphi=0$ on $D$, then $\left\{A^{\varphi}=0\right\} \supset\{\mathcal{R} \subset \bar{D}\} P_{\mu}$-a.s. and $\left\{A^{\varphi}=0\right\} \supset$ $\left\{\mathcal{R}^{*} \subset \bar{D}\right\} P_{\mu}$-a.s. for all $\mu \notin \mathcal{N}$.

\footnotetext{
${ }^{13}$ As in the case of "perfection", these theorems can be easily extended to functionals with an exceptional set.

${ }^{14} \mathrm{An}$ additive functional $A$ is natural if the process $A_{t+}$ is predictable (cf. [4,IV.61] or the Appendix to [9]). We believe that functional $A$ constructed in Theorem 1.3 is natural but this is not proved in the present paper.
} 
4.4. Proof of Theorem 1.4. This proof is similar to that of Theorem 3.3 in [18]. Let, for instance, $\Gamma \subset E$ be a compact $\mathcal{R}$-polar set. Put

$$
D_{n}=\left\{x \in E: d(x, \Gamma)>\frac{1}{n}\right\}
$$

where $d$ is the distance in the Martin space $\hat{E}$. Bounded positive continuous functions

$$
\varphi_{n}(x)=(1-n d(x, \Gamma))_{+}
$$

vanish on $D_{n}$. Consider the corresponding localizations $A^{\varphi_{n}}$. For every $\mu \notin \mathcal{N}$,

$$
A^{1} \geq A^{\varphi_{1}} \geq \cdots \geq A^{\varphi_{n}} \geq \cdots, \quad P_{\mu} \text {-a.s. }
$$

by $4.3 . \mathrm{B}$ and

$$
\left\{\mathcal{R} \subset D_{n}\right\} \subset\left\{A_{\infty}^{\varphi_{n}}=0\right\}, \quad P_{\mu} \text {-a.s. }
$$

by 4.3.C. Let $\mu(\Gamma)=0$. Since $\Gamma$ is $\mathcal{R}$-polar, $1_{\mathcal{R} \subset D_{n}} \uparrow 1 P_{\mu}$-a.s. and therefore $A_{\infty}^{\varphi_{n}} \rightarrow 0 P_{\mu}$-a.s. By the dominated convergence theorem,

$$
\lim P_{\mu} A_{\infty}^{\varphi_{n}}=0
$$

On the other hand, by 4.3.A,

$$
\begin{aligned}
P_{\mu} A_{\infty}^{\varphi_{n}} & =\int \mu(d x) \int_{E} g(x, y) \varphi_{n}(y) \eta(d y) \\
& +\int \mu(d x) \int \mu(d x) \int_{E^{*}} k(x, y) \varphi_{n}(y) \nu(d y) \downarrow \int \mu(d x) \int_{\Gamma} g(x, y) \eta(d y) .
\end{aligned}
$$

In combination with (4.9), this implies $\eta(\Gamma)=0$. The case of $\mathcal{R}^{*}$-polar set $\Gamma \subset E^{*}$ can be treated in a similar way.

\section{REFERENCES}

1. D. R. Adams and L. I. Hedberg, Function Spaces and Potential Theory, forthcoming book.

2. P. Baras and M. Pierre, Singularités éliminable pour des équations semi-linéares, Ann. Inst. Fourier, Grenoble 34 (1984), 185-206. MR 86j:35063

3. G. Choquet, Theory of capacities, Ann. Inst. Fourier 5 (1953-54), 131-295. MR 18:295g

4. C. Dellacherie and P.-A. Meyer, Probabilités et potentiel, Hermann, Paris, 1975, 1980, 1983, 1987. MR 58:7757, 82b:60001, 86b:60003, 89j:60001

5. E. B. Dynkin, Functionals of trajectories of Markov stochastic processes, Doklady Akademii Nauk SSSR 104:5 (1955), 691-694. MR 17:501b

6. __ Markov Processes, Springer-Verlag, Berlin, Göttingen and Heidelberg, 1965. MR 33:1887

7. _ Exit space of a Markov process, [English translation: Russian Math. Surveys, 24, 4, pp. 89-157.], Uspekhi Mat. Nauk 24,4 (148), 89-152. MR 41:9359

8. $ـ$ Superprocesses and partial differential equations, Ann. Probab. 21 (1993), 1185-1262. MR 94j:60156

9. __ An Introduction to Branching Measure-Valued Processes, American Mathematical Society, Providence, Rhode Island, 1994. MR 94:14

10. _ Minimal excessive measures and functions, [Reprinted in: E. B. Dynkin, Markov Processes and Related Problems of Analysis, London Math. Soc. Lecture Note Series 54, Cambridge University Press, Cambridge, 1982.], Trans. Amer. Math. Soc. 258 (1980), 217244. MR 81a:60086 
11. (1989), 255-282. MR 89k:60124

12. , A probabilistic approach to one class of nonlinear differential equations, Probab. Th. Rel. Fields 89 (1991), 89-115. MR 92d:35090

13. _ Branching particle systems and superprocesses, Ann. Probab. 19 (1991), 1157- 1194. MR 92j:60101

14. _ Path processes and historical processes, Probab. Th. Rel. Fields 90 (1991), 89-115. MR 92i:60145

15. _ Additive functionals of superdiffusion processes, Random walks, Brownian Motion and Interacting Particle Systems, Progress in Probability (Rick Durrett, Harry Kesten, eds.), vol. 28, Birkhäuser, Boston, Basel and Berlin, 1991. MR 93d:60122

16. _ Superdiffusions and parabolic nonlinear differential equations, Ann. Probab. 20 (1992), 942-962. MR 93d:60124

17. E. B. Dynkin and S. E. Kuznetsov, Superdiffusions and removable singularities for quasilinear partial differential equations, Comm. Pure \& Appl. Math (1996) (to appear).

18. E. B. Dynkin, S. E. Kuznetsov, Solutions of Lu $=u^{\alpha}$ dominated by L-harmonic functions, Journale d'Analyse (1996) (to appear).

19. A. Friedman, Partial Differential Equations of Parabolic Type, Prentice-Hall, Englewood Cliffs, N. J., 1964. MR 31:6062

20. M. Fukushima, Dirichlet Forms and Markov Processes, Kodansha, North-Holland, 1980. MR 81f: 60105

21. D. Gilbarg and N. S. Trudinger, Elliptic partial differential equations of second order, SpringerVerlag, Berlin and Heidelberg, 1983. MR 86c:35035

22. A. Gmira and L. Véron, Boundary singularities of solutions of some nonlinear elliptic equations, Duke Math. J. 64 (1991), 271-324. MR 93a:35053

23. J.-F. Le Gall, The Brownian snake and solutions of $\Delta u=u^{2}$ in a domain, preprint (1994).

24. C. Miranda, Partial Differential Equations of Elliptic Type, 2nd ed., Springer-Verlag, Berlin and Heidelberg, New York, 1970. MR 44:1924

25. M. Sharpe, General theory of Markov processes, Academic Press, San Diego, 1988. MR 89m:60169

Department of Mathematics, Cornell University, Ithaca, New York 14853-7901

E-mail address: ebd1@cornell.edu

Central Economics and Mathematical Institute, Russian Academy of Sciences, 117418, Moscow, Russia

Current address: Department of Mathematics, Cornell University, Ithaca, New York 148537901

E-mail address: sk47@cornell.edu 